\title{
The AGN fraction of submm-selected galaxies and contributions to the submm/mm-wave extragalactic background light
}

S. Serjeant ${ }^{1}$, M. Negrello ${ }^{1}$, C. Pearson ${ }^{1,2,17}$, A. Mortier ${ }^{3}$, J. Austermann ${ }^{4}$, I. Aretxaga ${ }^{5}$, D. Clements ${ }^{3}$, S. Chapman ${ }^{18}$, S. Dye ${ }^{6}$, J. Dunlop 7 , L. Dunne ${ }^{8}$, D. Farrah ${ }^{9}$, D. Hughes ${ }^{5}$, H.-M. Lee ${ }^{12}$, H. Matsuhara ${ }^{13}$, E. Ibar ${ }^{10}$, M. Im ${ }^{12}$, W.-S. Jeong ${ }^{16}$, S. Kim ${ }^{11}$, S. Oyabu ${ }^{13}$, T. Takagi ${ }^{13}$, T. Wada ${ }^{13}$, G. Wilson ${ }^{14}$, M. Vaccari ${ }^{15}$, and M. Yun ${ }^{14}$

${ }^{1}$ Department of Physics \& Astronomy, The Open University, Milton Keynes MK7 6BJ, UK

2 Rutherford Appleton Laboratory, Chilton, Didcot, Oxfordshire OX11 0QX, UK

3 Astrophysics Group, Dept. of Physics, Imperial College London, Prince Consort Road, London SW7 2BW, UK

${ }^{4}$ Center for Astrophysics and Space Astronomy, University of Colorado, Boulder, CO80309, USA

5 Instituto Nacional de Astrofísica, Optica y Electrónica (INAOE), Tonantzintla, Puebla, México

${ }^{6}$ Cardiff University, School of Physics \& Astronomy, Queens Buildings, The Parade, Cardiff CF24 3AA, UK

7 Scottish Universities Physics Alliance (SUPA), Institute for Astronomy, The University of Edinburgh, Royal Observatory, Blackford Hill, Edinburgh EH9 3HJ, UK

8 School of Physics \& Astronomy, University of Nottingham, University Park, Nottingham, NG7 2RD, UK

9 Astronomy Centre, University of Sussex, Falmer, Brighton BN1 9QH, UK

${ }^{10}$ UK Astronomy Technology Centre, Royal Observatory, Edinburgh EH9 3HJ, UK

11 Astronomy \& Space Science Department, Sejong University, 143-747 Seoul, South Korea

12 Department of Physics and Astronomy, Seoul National University, Shillim-Dong, Kwanak-Gu Seoul 151-742, South Korea

13 Institute of Space and Astronautical Science, Japan Aerospace Exploration Agency, Sagamihara, Kanagawa 229 8510, Japan

14 Department of Astronomy, University of Massachusetts, Amherst, MA01003, USA

15 Department of Astronomy, University of Padova, vic. Osservatorio 2, 35122 Padova, Italy

16 Space Science Divison, KASI, 61-1, Whaam-dong, Yuseong-gu, Deajeon 305-348, South Korea

17 Department of Physics, University of Lethbridge, 4401 University Drive, Lethbridge, Alberta T1J 1B1, Canada

18 Institute of Astronomy, University of Cambridge, Madingley Road, Cambridge CB3 OHA, UK

Received 15 October 2009 / Accepted 18 February 2010

\section{ABSTRACT}

\begin{abstract}
We present a comparison of the SCUBA half degree extragalactic survey (SHADES) at $450 \mu \mathrm{m}, 850 \mu \mathrm{m}$ and $1100 \mu \mathrm{m}$ with deep guaranteed time $15 \mu \mathrm{m}$ AKARI FU-HYU survey data and Spitzer guaranteed time data at 3.6-24 $\mu \mathrm{m}$ in the Lockman hole east. The AKARI data was analysed using bespoke software based in part on the drizzling and minimum-variance matched filtering developed for SHADES, and was cross-calibrated against ISO fluxes. Our stacking analyses find AKARI $15 \mu \mathrm{m}$ galaxies with $\gtrsim 200 \mu \mathrm{Jy}$ contribute $>10 \%$ of the $450 \mu \mathrm{m}$ background, but only $<4 \%$ of the $1100 \mu \mathrm{m}$ background, suggesting that different populations contribute at mm-wavelengths. We confirm our earlier result that the ultra-deep $450 \mu \mathrm{m}$ SCUBA-2 cosmology survey will be dominated by populations already detected by AKARI and Spitzer mid-infrared surveys. The superb mid-infrared wavelength coverage afforded by combining Spitzer and AKARI photometry is an excellent diagnostic of AGN contributions, and we find that (23-52)\% of submmselected galaxies have AGN bolometric fractions $f_{\mathrm{AGN}}>0.3$.
\end{abstract}

Key words. galaxies: starburst - infrared: galaxies - galaxies: active - galaxies: statistics

\section{Introduction}

The pioneering submm-wave observations of the Hubble deep field north (Hughes et al. 1998; Barger et al. 1998) and galaxy cluster gravitational lenses (Smail et al. 1997) revealed the presence of completely unanticipated populations of submmluminous galaxies with fluxes of several mJy at $850 \mu \mathrm{m}$. This discovery was one of the first in a series that led to the development and ultimately wide acceptance of "downsizing" phenomenological models of galaxy evolution, that massive galaxies formed the bulk of their stars at high redshifts in large starburst events, with the opposite behaviour found in lowermass systems. This is contrary to naive expectations from hierarchical models, but nevertheless it was found these observations could be made consistent with semi-analytic hierarchical models by adjusting the physical parameters used to characterised feedback processes (e.g. Granato et al. 2006) and/or with adjustments to the initial mass function (e.g. Baugh et al. 2005; Lacey et al. 2008).

Indeed the existence of strong relationships between supermassive black hole masses in nearby galaxies and the properties of the host bulges or spheroids (e.g. the Magorrian relation, Magorrian et al. 1998; see also e.g. Ferrarese \& Merritt 2000) all but require a strong link between high-redshift black hole accretion and the generation of the stellar mass of the host. 
This is reinforced by the observations that many or perhaps all quasars show evidence of star formation (e.g. Lehnert et al. 1992; Hughes et al. 1997; Aretxaga et al. 1998; Brotherton et al. 1999; Lutz et al. 2008; Mullaney et al. 2010; Shi et al. 2009; Veilleux et al. 2009; Serjeant \& Hatziminaoglou 2009). While submm and mm-wave direct detections are only made in a minority of high-redshift quasars (e.g. Carilli et al. 2001; Omont et al. 2001; Isaak et al. 2002; Bertoldi \& Cox 2002; Omont et al. 2003; Priddey et al. 2003a,b; Willlot et al. 2003; Bertoldi et al. 2003), stacking the far-infrared to mm-wave non-detections yielded significant detections and demonstrated clearly that all quasars are on average ultraluminous starbursts (Serjeant \& Hatziminaoglou 2009). If submm-selected galaxies are the violent starbursts that mark the sites of the progenitors of presentday giant ellipticals, then the presence and nature of AGN in submm-selected galaxies may track the feedback processes regulating the stellar mass assembly.

Are the bolometric luminosities of submm galaxies dominated by the release of gravitational binding energy around a central supermassive black hole, or are they dominated by the release of nuclear binding energy in starbursts? Similar questions were asked of local ultraluminous infrared galaxies in the 1990s (see e.g. Genzel \& Cezarsky 2000); now the issues of feedback and the Magorrian relation add an additional perspective and motivation to the higher-redshift equivalent questions for submm-selected galaxies. Deep 2-10 keV imaging of submmselected galaxies has found that around three-quarters show evidence of an active nucleus (Alexander et al. 2005), the majority of which are heavily obscured. Nevertheless, the X-ray to far-infrared luminosity ratios suggest that star formation rather than active nuclei typically dominate the bolometric output, assuming the underlying spectral energy distributions of the active nuclei resemble those of unobscured quasars. In order to eliminate the possibility that Compton-thick active nuclei in submmselected galaxies have different underlying spectral energy distributions than the comparator populations, one should sample the spectra around the expected peak of the dust torus output, in the mid-infrared.

The advent of the Spitzer space telescope led to the discovery of new dust-shrouded galaxy populations with starbursts and/or active nuclei (e.g. Martínez-Sansigre et al. 2005; Dey et al. 2008a,b). The mid-IR where the dust torus luminous output peaks is the ideal place to test for AGN; we expect starforming galaxies to have strong $\mathrm{PAH}$ and silicate features, while AGN dust tori have much more featureless spectra, with the possible sole exception of a $10 \mu \mathrm{m}$ rest-frame silicate absorption trough. We found in Negrello et al. (2009) that with sufficiently comprehensive broad-band photometry in the mid-IR, starburst galaxies can have photometric redshift determinations from the redshifting of PAH features. Furthermore, if the bolometric AGN fraction is more than $30 \%$, the mid-IR SEDs are sufficiently close to featureless power-laws in broad-band photometry that photometric redshifts are impossible with mid-IR data alone. However in these cases, the power-law SEDs are a useful signature of bolometrically-significant AGN regardless of redshift. In this case, the longer wavelength range obtained by combining AKARI and Spitzer offers significant advantages over the insights available (albeit useful ones) from broad-band Spitzer photometry alone (e.g. Yun et al. 2008). A recent mid-infrared spectroscopic study of 13 submm-selected galaxies (Pope et al. 2008) found only $2 / 13$ with such power-law mid-infrared spectral energy distributions; similarly, Menéndez-Delmestre et al. (2009) found 4/23 submm-selected galaxies with continuumdominated mid-infrared spectra. Clearly there is a need for larger samples of submm-selected galaxies with constrained AGN bolometric fractions.

Submm galaxies are also significant contributors to the submm-wave extragalactic background light (EBL). The EBL can be expressed as an integral over the comoving volumeaveraged luminosity density (with an additional $(1+z)$ factor, e.g. Peacock 1999), so at any redshift $z$ the galaxies that dominate the far-IR to mm-wave EBL are also the ones that dominate the star formation rate at that $z$. But resolved submm galaxies only contribute a few tens of percent at most (Hughes et al. 1998) to the EBL at those wavelengths. To constrain the populations that contribute the rest one needs to use stacking analyses. Several populations of galaxies have been shown to contribute significant minorities of the submm EBL, such as EROs and HEROs, BzK galaxies, (e.g. Coppin et al. 2004; Takagi et al. 2007; Greve et al. 2009), and Lyman-break galaxies (e.g. Peacock et al. 2000). In Serjeant et al. (2008) we found that the $24 \mu \mathrm{m}$-selected galaxies contribute the bulk of the $450 \mu \mathrm{m}$ background, but that at $850 \mu \mathrm{m}$ there are unknown new populations that contribute the bulk of that background.

In this paper we will constrain the AGN bolometric fractions of a sample of submm-selected galaxies in the Lockman hole east, taken from the SCUBA half degree extragalactic survey (SHADES), using mid-infrared photometry from the AKARI and Spitzer space telescopes. We also constrain the contributions the mid-infrared populations make to the submm and mm-wave extragalactic background light using stacking analyses. SHADES Papers I and II (Mortier et al. 2005; Coppin et al. 2006) present the SHADES survey design, data analysis and source counts. Paper III (Ivison et al. 2007) gave radio and mid-infrared counterparts of SHADES galaxies. SHADES Paper IV (Aretxaga et al. 2007) presents photometric redshift constraints of submm galaxies derived from far-infrared and radio data alone. SHADES Paper V (Takagi et al. 2007) constrains the submm properties of near-infrared-selected galaxies. Paper VI (Coppin et al. 2007) gave $350 \mu \mathrm{m}$ photometry for SHADES galaxies, while Papers VII and VIII (Dye et al. 2008a,b; and Clements et al. 2008, respectively) analysed the multiwavelength spectral energy distributions based on data available at the time. SHADES Paper IX (Serjeant et al. 2008) made submm stacking analyses of ISO and Spitzer-selected galaxies, and in this paper we will extend this analysis to include the AKARI catalogues described in the Sect. 2. Austermann et al. (2010) extended the SHADES survey to mm-wavelengths and substantially increased the areal coverage. The comparison of the $850 \mu \mathrm{m}$ and $1100 \mu \mathrm{m}$ data, including a critical comparison of the $1100 \mu \mathrm{m}$ data from different instruments and a systematic search for populations of submm-wave drop-outs, will be given in Negrello et al. (2009, in preparation). In this paper we will use the AzTEC $1100 \mu \mathrm{m}$ data, rather than the $1100 \mu \mathrm{m}$ data from Coppin et al. (2008) which has not been corrected for Eddington bias (Eddington 1913). We will also use the multi-wavelength data from SHADES Papers III, VI, VII and VIII (Ivison et al. 2007; Coppin et al. 2007; Dye et al. 2008a,b; and Clements et al. 2008, respectively) who presented spectral energy distribution (SED) fits of SHADES galaxies.

\section{Data acquisition}

\subsection{Submm and $\mathrm{mm}$-wave observations}

The SHADES was the largest project on the James Clerk Maxwell Telescope from 2003-2005. During the instrument lifetime of SCUBA the survey mapped a total of approximately 
a quarter of a square degree at $850 \mu \mathrm{m}$ to a typical $3.5 \sigma$ depth of $8 \mathrm{mJy}$, in the Lockman hole east and the Subaru-XMM deep field (Mortier et al. 2005). The originally-planned areal coverage and more was completed by the SHADES team with the AzTEC instrument at $1100 \mu \mathrm{m}$ (Austermann et al. 2010) to typical $3.5 \sigma$ depths in the Lockman hole east of 3.1-4.6 mJy. Both SHADES fields benefit from enormous multi-wavelength wide-field campaigns, and the Lockman hole east in particular has some of the deepest Spitzer Space Telescope mid-infrared mapping of any contiguous field over hundreds of square arcminutes. More details on the SHADES project, including the survey goals, methodology and submm data analysis, can be found in SHADES Papers I and II (Mortier et al. 2005; Coppin et al. 2006). Details on the SHADES mm-wave data analysis are given in Austermann et al. (2010). We use the Austermann et al. (2010) mm-wave measurements in preference to Coppin et al. (2008), since the former but not the latter have been corrected for Eddington bias.

\subsection{Mid-infrared observations}

The Lockman hole east was targetted by the Spitzer space telescope in guaranteed time. The field was mapped with the IRAC and MIPS instruments (Fazio et al. 2004; Rieke et al. 2004) and sources extracted to depths of $4.47 \mu \mathrm{Jy}$ at $3.6 \mu \mathrm{m}(3 \sigma), 4.54 \mu \mathrm{Jy}$ at $4.5 \mu \mathrm{m}(3 \sigma), 20.9 \mu \mathrm{Jy}$ at $5.8 \mu \mathrm{m}(3 \sigma), 12.5 \mu \mathrm{Jy}$ at $8 \mu \mathrm{m}(3.2 \sigma)$ and $38 \mu \mathrm{Jy}$ at $24 \mu \mathrm{m}(4 \sigma)$. For more details of this catalogue, see Serjeant et al. (2008, and references therein). The Lockman hole east was also mapped at $15 \mu \mathrm{m}$ with the CAM instrument on the ISO. More details of the ISO survey can be found in Elbaz et al. (1999) and Rodighiero et al. (2004).

The Lockman hole east was also the subject of a $10^{\prime} \times 30^{\prime}$ survey in the L15 $15 \mu \mathrm{m}$ filter by infrared camera (IRC) of AKARI, as part of the $F U-H Y U$ mission programme (Pearson et al. 2010), to a typical $1 \sigma$ noise level of $20 \mu \mathrm{Jy}$. This programme targetted well-studied Spitzer fields to capitalize on the comprehensive multi-wavelength data sets available in these fields. Some $F U-H Y U$ observations in the GOODS-N field have been presented in Negrello et al. (2009).

A pipeline analysis of the IRC is available in the Image Reduction and Analysis Facility (IRAF). Despite being an excellent general-purpose analysis, we found that the processing was not optimal for our purposes of minimum-variance point source extraction. The standard pipeline locates and flags bad pixels, then interpolates the image to correct for field distortions. However this interpolation stage creates Moiré effects, correlates the noise between pixels and in some cases spreads bad individual pixel readouts over several pixels in the interpolated image. Furthermore, some of the AKARI observations had been mistakenly taken without the requested dithering intended to remove bad pixels, so our only option was to use the spacecraft jitter, which necessarily requires a bespoke analysis.

We had previously developed mapping and minimumvariance point source extraction tools in the Interactive Data Language (IDL) for the SHADES survey, so we opted to use a similar approach here. We stopped the IRC pipeline after the flat fielding, imported the data into IDL, then determined an approximate pointing solution using catalogues of ISO and Spitzer sources in the field. The relative jitter was found by measuring centroids of bright sources in each data frame, and hence we could determine a World Coordinate System solution for each frame. Bad pixels were identified and masked using the same pixel histogram fitting procedure employed in the SCUBA Hubble deep field north and SHADES observations
(Serjeant et al. 2003a; Mortier et al. 2005). Several frames were affected by Earthshine, so we performed a polynomial fit to each row, then each column, then identified and corrected a small number of pixels which reported consistently low counts regardless of target. This latter effect may indicate the need to improve the flat field determinations, but the offsets induced by these corrections are much smaller than our photometric errors. Following the best practice in SCUBA observations we determined the noise in each detector pixel using Gaussian fits to readout histograms. We adopted the field distortion corrections as coded in the IRAF IRC pipeline, but from a comparison of our initial AKARI catalogues against Spitzer observations we found evidence for further field distortions. We fit to these using a second-order polynomial and incorporated the correction into our total field distortion solution.

To mosaic the individual frames onto a master image we used the "zerofootprint" drizzling technique developed originally for SCUBA (Serjeant et al. 2003a): detector pixels are mapped onto much finer sky pixels, accounting for field distortion and jitter, with the flux from a detector pixel deposited in a single sky pixel. Where a sky pixel has multiple observations, fluxes are combined using noise-weighted coadds. The final coadded map represents a detector pixel's view of the sky, in the sense that at every point it reports the noise-weighted mean flux of all detector pixels centred exactly on that point. Notwithstanding crosstalk between detector pixels (which we neglect), the final coadded map also has statistically independent pixels, i.e. without covariances.

We performed a further deglitching stage on the coadded map. The minimum-variance estimator for the point source flux $F$ at any point in the map can be expressed a noise-weighted point spread function convolution, as used in submm surveys (Serjeant et al. 2003a; Mortier et al. 2005):

$F=\frac{(I W) \otimes P}{W \otimes P^{2}}$

where $I$ is the coadded image, $W$ is the weight image (the reciprocal of the noise squared), and $P$ is the point spread function. Propagating errors on Eq. (1) yields an expression for $\Delta F$, the error on $F$ :

$(\Delta F)^{2}=\frac{1}{W \otimes P^{2}}$.

For the point spread function we assumed a Gaussian with a full width half maximum of 5.47".

Our point source detection map is the $F / \Delta F$ image, in which we found objects using a connected pixels algorithm. Objects were identified as discrete regions with map values greater than a given threshold. We used a series of thresholds of 3-10 $\sigma$ in steps of $1 \sigma$, because objects which are blended at a low threshold may be distinct objects at a brighter threshold (e.g. Mortier et al. 2005), yielding a catalogue of 622 distinct objects. The IRC pipeline flux calibration is given only for photometry taken with the IRC pipeline outputs, which is necessarily systematically different to our pipeline outputs, so we cross-matched our catalogue with the previous ISO $15 \mu \mathrm{m}$ sources from Rodighiero et al. (2004) and based our calibration on the cross-matchings so the average flux ratio is unity, shown in Fig. 1. The final AKARI $15 \mu \mathrm{m}$ sources are shown in Figs. 2 and 3.

\section{Methods}

For the stacking analyses of submm galaxies we adopted the same methodologies of Serjeant et al. (2008). In brief, we first 


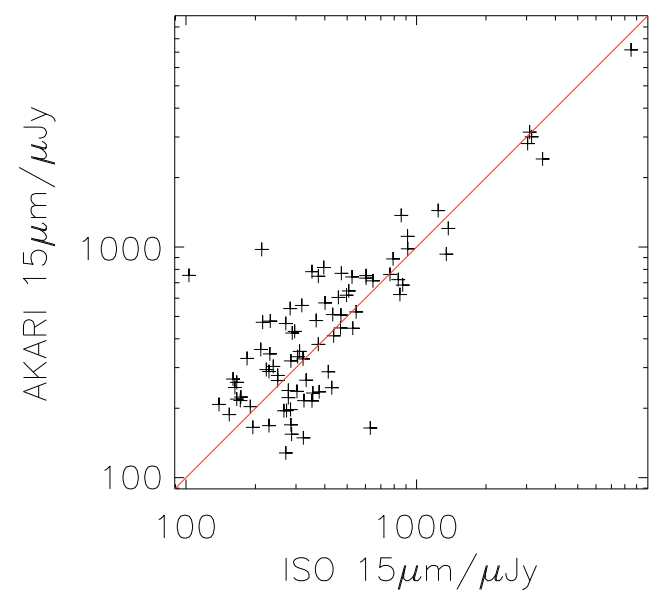

Fig. 1. Comparison of $15 \mu \mathrm{m}$ fluxes obtained in the Lockman hole east with the ISO (Elbaz et al. 1999; Rodighiero et al. 2004) with those obtained with our AKARI survey (Pearson et al. 2010). Note that the mean flux calibration of AKARI has been determined by the cross-matching with the ISO survey (implicitly using the ISO flux calibration) and is not determined independently in this study.

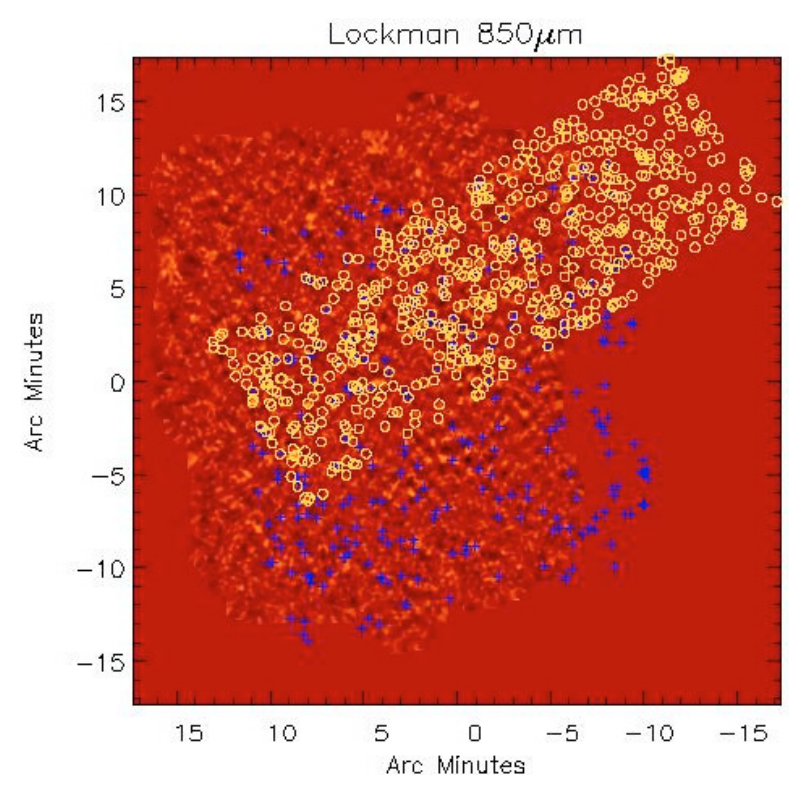

Fig. 2. The SHADES SCUBA $850 \mu \mathrm{m}$ signal-to-noise image of the Lockman hole east. The AKARI $15 \mu \mathrm{m}$ sources are overplotted in yellow (rectangular coverage) and the ISO $15 \mu \mathrm{m}$ sources are overplotted in blue (approximately square coverage).

subtract the catalogued objects from the $850 \mu \mathrm{m}$ and $1100 \mu \mathrm{m}$ maps (there are no secure $450 \mu \mathrm{m}$ point source detections). We used Gaussian point spread functions for the submm data and the point spread function in Austermann et al. (2010) for the AzTEC data. We refer to these maps as the residual maps. The comparison of the histograms of mm-wave fluxes in the map and at the positions of $15 \mu \mathrm{m}$-selected galaxies yielded a possibly strong signal $(\sim 8 \sigma)$ but which appeared to be dominated by a non-Gaussian shape to the latter histogram (Fig. 4). This could be suggestive of weak but coherent features in parts of the map, but which would necessarily have to have absolute values less than $1 \sigma$ per beam, making investigation difficult. Alternatively, this could be a consequence of having a relatively small number of AzTEC beams in the $15 \mu \mathrm{m}$ survey coverage. Without passing

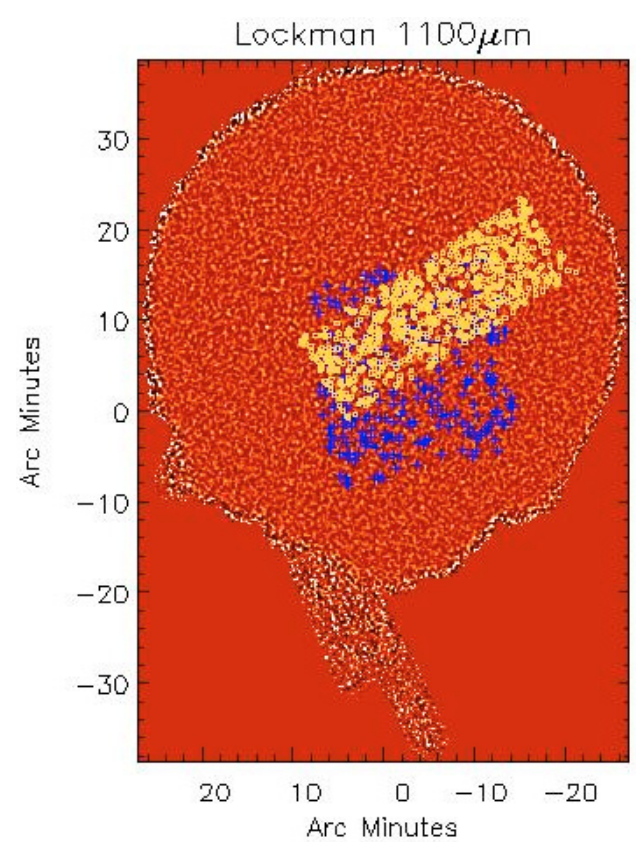

Fig. 3. The SHADES AzTEC $1100 \mu$ m image of the Lockman hole east. The AKARI $15 \mu \mathrm{m}$ sources are overplotted in yellow (rectangular coverage) and the ISO $15 \mu \mathrm{m}$ sources are overplotted in blue (approximately square coverage).

judgement on these issues, we have opted to take a very conservative approach and adopted an additional beamswitching sky subtraction, by subtracting from the AzTEC residual map the same map offset by 100 pixels East, or 300". We also derived a noise map for this beamswitched residual map by measuring the standard deviation in a $45^{\prime \prime}$ radius circular region at every point in the map.

There is some disagreement in the literature over the best practice in stacking methodology Some (such as Peacock et al. 2000; Serjeant et al. 2004, 2008) have opted to subtract known point sources, while others (such as Wang et al. 2007; Scott et al. 2008; Marsden et al. 2009) opt to use the unsubtracted map. In essence, it depends on what one wants to measure. If one requires the total intensity from all galaxies, one should not subtract any flux (or the subtracted population should be added afterwards). If however one requires the mean flux from a particular population, as distinct from all galaxies as a whole, then one needs to clean the map to avoid any biases in the mean.

In Serjeant et al. (2008) we found that the submm-selected galaxies had very different mid-IR/submm flux ratios to the galaxies selected at $24 \mu \mathrm{m}$. This could be due to bimodality, i.e. two populations, or we could be sampling two ends of a continuum; it is difficult to distinguish these possibilities from stacks alone. The possibility that a few $15 \mu \mathrm{m}$-selected galaxies are (relative to the rest) pathological would suggest the use of point-source-subtracted maps, which is the approach we have adopted.

We take unweighted mean averages of the submm fluxes in the residual maps (or for the AzTEC observations, mm-wave fluxes) at the positions of mid-infrared-selected galaxies. By the central limit theorem the unweighted mean flux is Gaussian distributed with a dispersion of $\sigma / \sqrt{N}$ where $\sigma$ is the standard deviation of the sample of fluxes and $N$ is the number of fluxes being averaged. We also use Kolmogorov-Smirnoff tests to compare the histogram of fluxes at the positions of mid-infrared-selected galaxies with the histogram of the submm/mm-wave fluxes in 

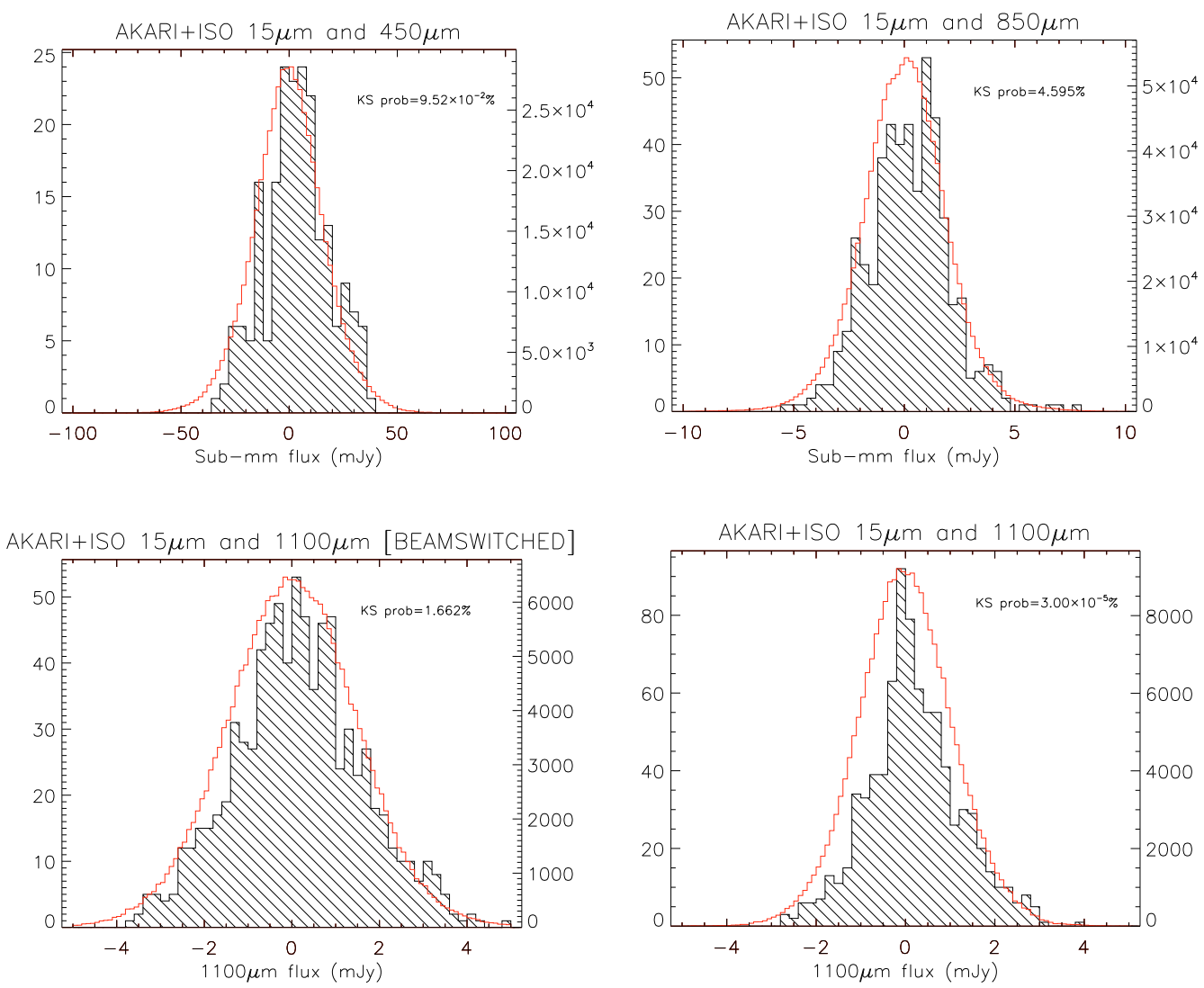

Fig. 4. Histograms of the submm and mm-wave fluxes in the regions with $15 \mu \mathrm{m}$ data (unhatched data, right-hand ordinates) compared to the submm and mm-wave flux measurements at the positions of $15 \mu \mathrm{m}$ point sources (hatched data, left-hand ordinates). The significance values of Kolmogorov-Smirnov tests are quoted in the figures. As noted in the text, the $1100 \mu \mathrm{m}$ map has had an additional sky subtraction from beamswitching.

the submm/mm-wave map as a whole, excluding regions with no mid-infrared data. This latter test inherently incorporates a control comparison. We include the $15 \mu \mathrm{m}$ survey from ISO (Elbaz et al. 1999; Rodighiero et al. 2004); although this survey is not as deep as our AKARI mapping, it increases the areal coverage of the stacking analysis (Figs. 2 and 3) and therefore also the number of submm and mm-wave beams contributing, which improve the stacked signal (Serjeant et al. 2008). Unweighted means give cosmetically poor results for stacked postage stamps due to edge effects, so we used noise-weighted means for the purposes of making stacked postage stamps only.

For the characterisation of the spectral energy distributions of submm-selected galaxies, we assume a minimum photometric error of ten percent. We use an ensemble of starburst model outputs from Takagi et al. $(2003,2004)$ and an ensemble of dust torus model outputs from Efstathiou \& Rowan-Robinson (1995), following the methodology of Negrello et al. (2009). We make linear superimpositions of these model spectral energy distributions neglecting the small effects of energy transfer between the dust torus and the starburst dust components. The key physical parameters in these models are: the torus opening angle $\theta$ (which we set to $45^{\circ}$ ), the ratio of outer and inner torus radii (which we set to 20), and viewing angle $\theta_{\text {view }}$; starburst compactness parameter $\Theta$, age $T$ and dust composition (Milky Way, SMC or LMC). A star formation timescale of $100 \mathrm{Myr}$ was adopted for the Takagi et al. models. A wider parameter space of AGN models is not justified by the contraints available from our data. We leave the redshift as a further free parameter even where spectroscopic redshifts have been published, to avoid dependence on the often-uncertain discussion of identifications. The total number of fitted parameters (including normalisation, but not counting dust composition) is three, and two more with the addition of AGN, plus a further fitting parameter for redshift.

\section{Results}

\subsection{Stacking analysis results}

Figure 5 shows noise-weighted stacked $450 \mu \mathrm{m}, 850 \mu \mathrm{m}$ and $1100 \mu \mathrm{m}$ postage stamps of the AKARI and ISO $15 \mu \mathrm{m}$-selected galaxies. This is a significant improvement over the detections in Serjeant et al. (2008), and for the first time extends the stacked signal of mid-infrared-selected galaxies to mm-wavelengths. The unweighted mean submm-wave and mm-wave fluxes of the $15 \mu \mathrm{m}$-selected galaxies are $\left\langle S_{450 \mu \mathrm{m}}\right\rangle=(3.7 \pm 1.1) \mathrm{mJy}$, $\left\langle S_{850 \mu \mathrm{m}}\right\rangle=(0.206 \pm 0.084) \mathrm{mJy}$ and $\left\langle S_{1100 \mu \mathrm{m}}\right\rangle=(0.148 \pm$ $0.052) \mathrm{mJy}$. The $450: 1100 \mu \mathrm{m}$ flux ratio is consistent with a grey-body index of $\beta=1.5$ on the Rayleigh-Jeans tail, though the $850 \mu \mathrm{m}$ stacked flux is marginally discrepant. In the source count model of Pearson (2005) the predicted median redshift of our sample is $z \sim 1$, with of the order $10 \%$ at $z>2$.

Figure 4 shows the histograms of submm and mm-wave fluxes at the positions of the $15 \mu \mathrm{m}$-selected galaxies, compared to the histograms of the maps as a whole were $15 \mu \mathrm{m}$ data is available. The probabilities that the $15 \mu \mathrm{m}$-selected galaxies are drawn randomly from the submm maps are $\sim 10^{-3}$ at $450 \mu \mathrm{m}$, 0.046 at $850 \mu \mathrm{m}$ and 0.016 at $1100 \mu \mathrm{m}$, i.e. a significant stacking detection. These probabilities in all three cases are due to 

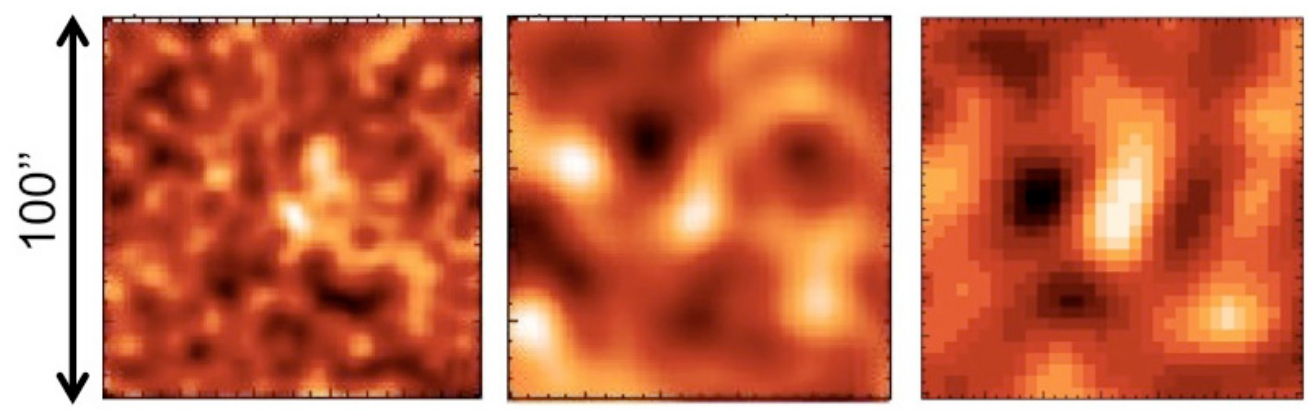

Fig. 5. Stacked mm-wave and submm signal-to-noise postage stamps of $15 \mu \mathrm{m}$ sources from AKARI and ISO, at (from left to right) $450 \mu \mathrm{m}$, $850 \mu \mathrm{m}$ and $1100 \mu \mathrm{m}$. The images are scaled from $-2.81 \sigma$ to $3.73 \sigma(450 \mu \mathrm{m}),-2.73 \sigma$ to $2.83 \sigma(850 \mu \mathrm{m})$ and $-3.01 \sigma$ to $2.99 \sigma(1100 \mu \mathrm{m})$.

slight asymmetries or positive shifts in the submm/mm-wave flux distributions.

\subsection{SED fitting results}

We found centroids in the $15 \mu \mathrm{m}$ AKARI point source detection map at the positions of SHADES Lockman galaxies reported in Dye et al. (2008a,b). Where no centroid solution could be obtained, we took the value of the point source flux $F$ and its associated error $\Delta F$ at the SHADES counterpart position (Eqs. (1) and (2)). Tables 1 and 2 give a compilation of available photometry of the SHADES galaxies from Dye et al. (2008a,b), Ivison et al. (2007) and Coppin et al. (2007), and incorporating our new $15 \mu \mathrm{m}$ photometry.

Following Negrello et al. (2009), we found the minimum $\chi^{2}$ solution for the available photometry assuming a mix of starburst and active nucleus, allowing the relative bolometric fractions of the components $\left(f_{\mathrm{SB}}\right.$ and $f_{\mathrm{AGN}}=1-f_{\mathrm{SB}}$ respectively) to vary. Table 3 gives the best-fit parameters of these two-component fits, excluding those for which no fit could be made, but including for completeness those for which the fitting is under-constrained. Figures 6 to 10 show the best-fit SEDs, as well as the best-fit starburst-only model for each SHADES galaxy. In many cases, adding the active nucleus is a requirement for reproducing the mid-infrared data. In several other cases however, no acceptable $\chi^{2}$ could be found, because the submm and mm-wave data exceeded the range given by the models. In these cases, a further cold cirrus component may contribute significantly to the longest wavelengths.

Several galaxies are worth discussing in detail. The galaxies with an excess at submm/mm-wavelengths are LOCK850.009, 011, 013, 031, 037B, 040, 043/043B, 053, 070, and 071. In addition, Lock850.076 has peculiar submm/mm-wave colours, perhaps due to incorrect flux deboosting in either the submm or mm-wave data. The $15 \mu \mathrm{m}$ flux in Lock850.003B may be contaminated by a nearby object. The SED fits in some cases suggest particular identifications, e.g. LOCK850.010B is preferred over LOCK850.010 (not plotted since no acceptable fit could be made to the data), as is LOCK850.015 over LOCK850.015B, LOCK850.035 over LOCK850.035B, and LOCK850.073 over LOCK850.073B. The identifications for LOCK850.004, LOCK850.009, LOCK850.043 and LOCK850.77 remain ambiguous.

\section{Discussion and conclusions}

Following the methodology of Serjeant et al. (2008), we can estimate the contribution that clustering of the mid-infrared population makes to the submm and mm-wave stacks, by integrating the correlation function $w(\theta)$ with respect to angle $\theta$ :

$S=I \int_{0}^{\infty} w(\theta) B(\theta) 2 \pi \theta \mathrm{d} \theta$

where $B$ is the submm or mm-wave beam and $I$ is the total background intensity. We argued in Serjeant et al. (2008) that the clustered contributions at $450 \mu \mathrm{m}$ and $850 \mu \mathrm{m}$ can be neglected. At $1100 \mu \mathrm{m} \mathrm{Eq.} \mathrm{(3)} \mathrm{gives} S=0.12\left(\theta_{0} / \operatorname{arcsec}\right)^{0.8}$ for an assumed $w(\theta)=\left(\theta / \theta_{0}\right)^{-0.8}$. The value of $\theta_{0}$ is not well-determined for the mid-infrared population at these flux densities (typically $\gtrsim 200 \mu \mathrm{Jy}$ ), but Oliver et al. (2004) measured $\theta_{0}=1.24^{\prime \prime}$ for the faintest $3.6 \mu \mathrm{m}$-selected populations (note that nearly all $15 \mu \mathrm{m}$-selected galaxies are detectable at $3.6 \mu \mathrm{m}$ with Spitzer in even the deepest $15 \mu \mathrm{m}$ exposures, e.g. Hopwood et al. 2009). To avoid introducing model-dependences in our quoted results, we opt not to correct for clustering in the stacked flux numbers quoted here. Nevertheless, the total background contributed by the $15 \mu \mathrm{m}$ population in this paper is considerably less than the integrated background, so the clustered contribution estimated by this methodology is a very conservative upper limit.

The observed $1100 \mu \mathrm{m}$ stacked signal of $\left\langle S_{1100 \mu \mathrm{m}}\right\rangle=$ $(0.148 \pm 0.052) \mathrm{mJy}$ at a $15 \mu \mathrm{m}$ source combined with our observed surface density of $4950 \pm 17015 \mu \mathrm{m}$ galaxies per square degree (Poisson errors) corresponds to a $1100 \mu \mathrm{m}$ background contribution of $(0.73 \pm 0.26) \mathrm{Jy} \mathrm{deg}^{-2}$. The estimated total background at $1100 \mu \mathrm{m}$ is around $18 \mathrm{Jy} \mathrm{deg}^{-2}$ (e.g. Gispert et al. 2000). Given the contribution from clustering, we can set an upper limit of $4 \%$ to the contributions from these $15 \mu \mathrm{m}$ selected galaxies to the $1100 \mu \mathrm{m}$ extragalactic background light. Our $15 \mu \mathrm{m}$-selected sample is not as deep as the $24 \mu \mathrm{m}$-selected population used by Serjeant et al. (2008), but following the same methodology as we have applied at $1100 \mu \mathrm{m}$, we estimate background contributions of $(1.02 \pm 0.42) \mathrm{Jy} \mathrm{deg}^{-2}$ at $850 \mu \mathrm{m}$ and $18.3 \pm 5.4 \mathrm{Jy} \mathrm{deg}^{-2}$ and $450 \mu \mathrm{m}$, corresponding to $(3 \pm 1) \%$ of

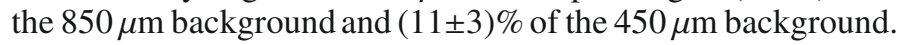
This is consistent with our results in Serjeant et al. (2008), implying the populations which dominate the ultra-deep confusionlimited maps expected with the SCUBA-2 instrument will overlap substantially with populations already detected with AKARI and Spitzer. 
S. Serjeant et al.: AGN fraction of submm-selected galaxies \& EBL

Table 1. Optical and near-infrared AB magnitude photometry of SHADES galaxies in the Lockman hole east.

\begin{tabular}{|c|c|c|c|c|c|c|c|c|}
\hline Name & $B(\mathrm{AB})$ & $R(\mathrm{AB})$ & $I(\mathrm{AB})$ & $z(\mathrm{AB})$ & $K(\mathrm{AB})$ & $3.6 \mu \mathrm{m}(\mathrm{AB}) 4.5 \mu \mathrm{m}(\mathrm{AB})$ & $5.8 \mu \mathrm{m}(\mathrm{AB})$ & $8 \mu \mathrm{m}(\mathrm{AB})$ \\
\hline Lock850.001 & & $26.29 \pm 0.1$ & $25.52 \pm 0.07$ & $25.7 \pm 0.18$ & & $22.31 \pm 0.3421 .77 \pm 0.29$ & & $21.78 \pm 1.12$ \\
\hline Lock850.002 & $26.75 \pm 0.1$ & $25.26 \pm 0.06$ & $24.8 \pm 0.06$ & $24.56 \pm 0.07$ & $21.85 \pm 0.28$ & $21.42 \pm 0.2521 .38 \pm 0.52$ & $21.29 \pm 0.45$ & \\
\hline Lock850.003 & $26.45 \pm 0.09$ & $24.9 \pm 0.06$ & $24.21 \pm 0.06$ & $23.47 \pm 0.06$ & $21.39 \pm 0.06$ & $20.66 \pm 0.21 \quad 20.67 \pm 0.2$ & $20.47 \pm 0.39$ & $21.09 \pm 0.44$ \\
\hline Lock850.003b & & & $25.56 \pm 0.07$ & $25.05 \pm 0.1$ & & $21.05 \pm 0.1921 .03 \pm 0.21$ & $20.9 \pm 0.44$ & $21.2 \pm 0.4$ \\
\hline Lock850.004 & $25.57 \pm 0$ & 24.48 & $24.17 \pm 0.06$ & $23.96 \pm 0.06$ & 21.8 & $20.83 \pm 0.2 \quad 20.49 \pm 0.19$ & 0.41 & $20.48 \pm 0.4$ \\
\hline Lock850.004b & $27.14 \pm 0.15$ & $26.09 \pm$ & $25.85=$ & $25.66 \pm$ & & 0.1920 .94 & 0.45 & \pm 0.43 \\
\hline 0.005 & & $25.66 \pm 0.06$ & $24.67 \pm 0.06$ & $23.77 \pm 0.06$ & $21.16 \pm 0.06$ & $20.62 \pm 0.2 \quad 20.61 \pm 0.21$ & $21.25 \pm$ & $21.53 \pm 0.33$ \\
\hline 0.006 & $28.08 \pm 0.34$ & $26.2 \pm 0.1$ & $25.81 \pm 0.08$ & $25.22 \pm 0.12$ & & $21.35 \pm 0.23 \quad 21.25 \pm 0.23$ & $20.93 \pm 0.58$ & $21.42 \pm 0.46$ \\
\hline
\end{tabular}

Lock850.008

$\begin{array}{lllllllllll}\text { Lock850.009 } & 26.86 \pm 0.11 & 25.53 \pm 0.06 & 24.79 \pm 0.06 & 24.43 \pm 0.06 & 21.58 \pm 0.06 & 20.25 \pm 0.2 & 20.23 \pm 0.22 & 20.43 \pm 0.54 & 20.91 \pm 0.45\end{array}$ Lock850.009b $24.04 \pm 0.0623 .09 \pm 0.0622 .3 \pm 0.06 \quad 21.8 \pm 0.06 \quad 20.42 \pm 0.06 \quad 19.83 \pm 0.1920 .26 \pm 0.1620 .56 \pm 0.5 \quad 21.21 \pm 0.46$ Lock850.010

Lock850.010b $26.2 \pm 0.06 \quad 25.67 \pm 0.0625 .09 \pm 0.0624 .31 \pm 0.06$

$22.71 \pm 0.4 \quad 22.27 \pm 0.35$

$\begin{array}{lllllllllllll}\text { Lock850.011 } & 26.91 \pm 0.12 & 26.09 \pm 0.09 & 25.37 \pm 0.06 & 24.63 \pm 0.07 & 22.17 \pm 0.11 & 21.53 \pm 0.19 & 21.56 \pm 0.18 & 22.11 \pm 0.5 & 21.57 \pm 0.41\end{array}$

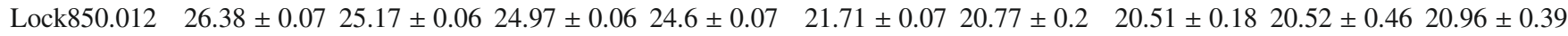

$\begin{array}{llllllllll}\text { Lock850.013 } & 21.71 \pm 0.06 & 20.67 \pm 0.06 & 20.37 \pm 0.06 & 20.31 \pm 0.06 & 19.64 \pm 0.06 & 20.22 \pm 0.2 & 20.56 \pm 0.16 & 21.08 \pm 0.41 & 20.56 \pm 0.35\end{array}$

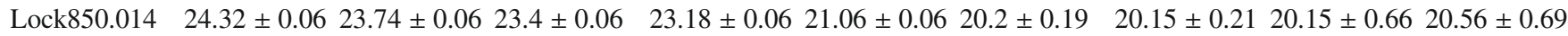

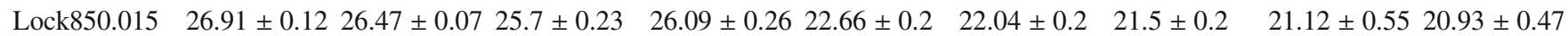

Lock850.015b

$\begin{array}{lllllllllllll}\text { Lock850.016 } & 27.28 \pm 0.17 & 25.2 \pm 0.06 & 23.99 \pm 0.06 & 23.47 \pm 0.06 & 20.45 \pm 0.06 & 19.93 \pm 0.21 & 19.65 \pm 0.22 & 19.73 \pm 0.35 & 20.37 \pm 0.31\end{array}$

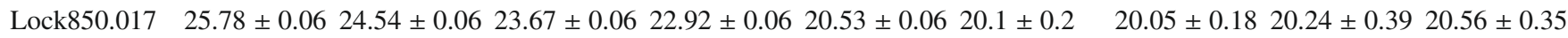

$\begin{array}{lll}\text { Lock850.017b } & 25.82 \pm 0.0725 .58 \pm 0.0724 .68 \pm 0.08\end{array}$

Lock850.018

Lock850.019

Lock850.021

Lock850.022

Lock850.023

Lock850.024

Lock850.026

Lock850.027

Lock850.028

Lock850.029

Lock850.030

Lock850.031

Lock850.033

Lock850.034

Lock850.035

Lock850.035b $24.65 \pm 0.0624 .31 \pm 0.0623 .83 \pm 0.0623 .64 \pm 0.0621 .74 \pm 0.0821 .51 \pm 0.2121 .33 \pm 0.2$

Lock850.036

Lock850.037

$\begin{array}{llllllllll}\text { Lock850.037b } 25.81 \pm 0.06 & 24.81 \pm 0.06 & 24.09 \pm 0.06 & 23.87 \pm 0.06 & 21.14 \pm 0.06 & 20.4 \pm 0.18 & 20.23 \pm 0.22 & 20.63 \pm 0.43 & 21.32 \pm 0.33\end{array}$

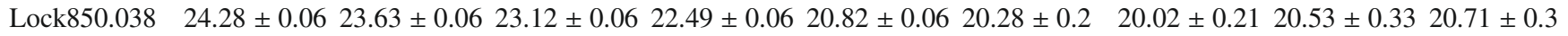

Lock850.039

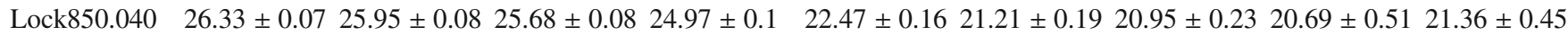

$\begin{array}{llllllllll}\text { Lock850.041b } 24.42 \pm 0.06 & 23.95 \pm 0.06 & 23.53 \pm 0.06 & 22.99 \pm 0.06 & 21.25 \pm 0.1 & 21.05 \pm 0.22 & 20.42 \pm 0.39 & 19.66 \pm 0.37\end{array}$

$\begin{array}{lllllllllllll}\text { Lock850.041 } & 22.95 \pm 0.06 & 22.05 \pm 0.06 & 21.53 \pm 0.06 & 21.05 \pm 0.06 & 19.96 \pm 0.06 & 19.27 \pm 0.16 & 19.26 \pm 0.19 & 19.53 \pm 0.4 & 19.69 \pm 0.34\end{array}$

$\begin{array}{llllllllllll}\text { Lock850.043 } & 26.21 \pm 0.06 & 25.18 \pm 0.06 & 24.82 \pm 0.06 & 24.48 \pm 0.06 & 21.6 \pm 0.07 & 21.19 \pm 0.2 & 21.07 \pm 0.19 & 20.88 \pm 0.53 & 21.52 \pm 0.42\end{array}$

$\begin{array}{llllllllll}\text { Lock850.043b } 23.66 \pm 0.3 & 23.01 \pm 0.15 & 22.46 \pm 0.11 & 21.82 \pm 0.06 & 20.14 \pm 0.06 & 19.69 \pm 0.16 & 19.52 \pm 0.18 & 19.78 \pm 0.36 & 20.39 \pm 0.31\end{array}$

$\begin{array}{llllllllll}\text { Lock850.047 } & 26.51 \pm 0.08 & 23.84 \pm 0.06 & 23.23 \pm 0.06 & 22.8 \pm 0.06 & 21.95 \pm 0.1 & 21.45 \pm 0.18 & 21.65 \pm 0.23 & 22.21 \pm 0.58\end{array}$

$\begin{array}{llllllllll}\text { Lock850.048 } & 23 \pm 0.06 & 21.47 \pm 0.06 & 21.11 \pm 0.06 & 20.62 \pm 0.06 & 19.63 \pm 0.06 & 19.73 \pm 0.18 & 20.07 \pm 0.21 & 20.53 \pm 0.39 & 19.41 \pm 0.32\end{array}$

$\begin{array}{llllllllllll}\text { Lock850.052 } & 26.17 \pm 0.06 & 24.56 \pm 0.06 & 23.61 \pm 0.06 & 22.88 \pm 0.06 & 20.39 \pm 0.06 & 19.3 \pm 0.2 & 19.64 \pm 0.19 & 20.25 \pm 0.39 & 20.71 \pm 0.3\end{array}$

$\begin{array}{lllllllll}\text { Lock850.052b } & 26.88 \pm 0.18 & 25.33 \pm 0.06 & 24.9 \pm 0.09 & 21.31 \pm 0.06 & 19.92 \pm 0.19 & 20.1 \pm 0.2 & 20.52 \pm 0.36 & 20.96 \pm 0.39\end{array}$

$\begin{array}{llllllllllll}\text { Lock850.053 } & 24.21 \pm 0.06 & 23.92 \pm 0.06 & 23.47 \pm 0.06 & 23.19 \pm 0.06 & 21.58 \pm 0.06 & 20.96 \pm 0.21 & 20.9 \pm 0.18 & 21.09 \pm 0.35 & 21.46 \pm 0.4\end{array}$

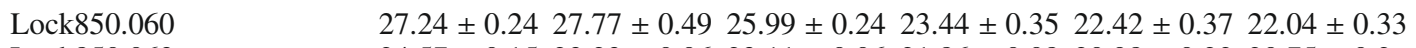

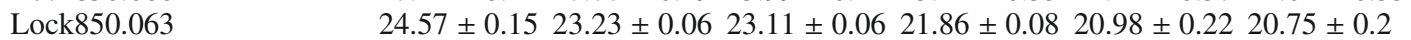

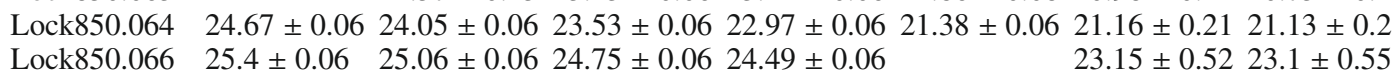

$\begin{array}{lllllllll}\text { Lock850.067 } & 28.41 \pm 0.3 & 28.4 \pm 0.5 & 26.58 \pm 0.14 & 25.64 \pm 0.14 & 22 \pm 0.31 & 21.73 \pm 0.3 & 22.38 \pm 0.77 & 22.32 \pm 0.68\end{array}$

$\begin{array}{llllllllll}\text { Lock850.070 } & 24.67 \pm 0.05 & 23.1 \pm 0.06 & 22.51 \pm 0.06 & 22.41 \pm 0.06 & 21.03 \pm 0.06 & 21.13 \pm 0.2 & 21.58 \pm 0.28 & 21.28 \pm 0.32\end{array}$

$\begin{array}{lllllllllll}\text { Lock850.071 } & 25.43 \pm 0.06 & 24.97 \pm 0.06 & 24.76 \pm 0.06 & 24.2 \pm 0.06 & 21.76 \pm 0.06 & 21.46 \pm 0.25 & 21.05 \pm 0.21 & 21.3 \pm 0.35\end{array}$

$\begin{array}{llllllllll}\text { Lock850.073 } & 24.35 \pm 0.06 & 23.63 \pm 0.06 & 23.14 \pm 0.06 & 22.67 \pm 0.06 & 20.65 \pm 0.06 & 20.33 \pm 0.2 & 20.22 \pm 0.18 & 20.36 \pm 0.42 & 20.59 \pm 0.36\end{array}$

$\begin{array}{llllllll}\text { Lock850.073b } & 24.94 \pm 0.06 & 24.43 \pm 0.06 & 24.07 \pm 0.06 & 23.61 \pm 0.06 & 21.79 \pm 0.08 & 20.73 \pm 0.19 & 20.78 \pm 0.43\end{array}$

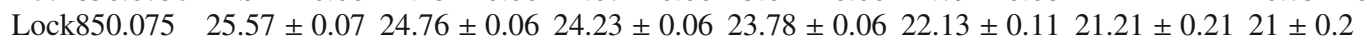

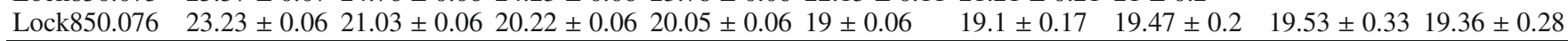


Table 1. continued.

\begin{tabular}{|c|c|c|c|c|c|c|c|c|c|}
\hline Name & $B(\mathrm{AB})$ & $R(\mathrm{AB})$ & $I(\mathrm{AB})$ & $z(\mathrm{AB})$ & $K(\mathrm{AB})$ & $3.6 \mu \mathrm{m}(\mathrm{AB})$ & $4.5 \mu \mathrm{m}(\mathrm{AB})$ & $5.8 \mu \mathrm{m}(\mathrm{AB})$ & $8 \mu \mathrm{m}(\mathrm{AB})$ \\
\hline Lock850.077 & $27.02 \pm 0.13$ & $26.5 \pm 0.13$ & $25.86 \pm 0.09$ & $24.81 \pm 0.08$ & & $22.23 \pm 0.34$ & $22.23 \pm 0.36$ & & \\
\hline Lock850.077b & $27.59 \pm 0.12$ & $24.97 \pm 0.06$ & $24.62 \pm 0.06$ & $23.44 \pm 0.06$ & $21.14 \pm 0.06$ & $20.12 \pm 0.2$ & $20.03 \pm 0.16$ & & \\
\hline Lock850.078 & $24.84 \pm 0.02$ & $24.39 \pm 0.06$ & $24.03 \pm 0.06$ & $23.84 \pm 0.06$ & $22.39 \pm 0.14$ & & & & \\
\hline Lock850.079 & $26.39 \pm 0.07$ & $26.6 \pm 0.14$ & $25.54 \pm 0.07$ & $25.05 \pm 0.1$ & $22.06 \pm 0.12$ & $21.04 \pm 0.2$ & $20.79 \pm 0.2$ & $20.65 \pm 0.51$ & $20.52 \pm 0.36$ \\
\hline 50.081 & $23.75 \pm 0.05$ & 23.18 & 22.65 & $22.19 \pm 0.06$ & 20.07 & .19 & $18.9 \pm 0.18$ & 18. & $17.2 \pm 0.19$ \\
\hline 50.083 & $21.27 \pm 0.06$ & 19.76 & & $18.96 \pm 0.06$ & 18. & 18. & $19.21 \pm 0.19$ & $19.81 \pm 0.21$ & $18.61 \pm 0.2$ \\
\hline 0.087 & $26.7 \pm 0.1$ & $25.58 \pm 0.07$ & $24.86 \pm 0.06$ & $24.72 \pm 0.08$ & $21.82 \pm 0.08$ & $20.63 \pm 0.21$ & $20.02 \pm 0.21$ & $20.1 \pm 0.39$ & $20.21 \pm 0.26$ \\
\hline Lock850.100 & $23.87 \pm 0.06$ & $23.43 \pm 0.06$ & $23.16 \pm 0.06$ & $22.76 \pm 0.06$ & $21.36 \pm 0.06$ & & & & \\
\hline
\end{tabular}

Notes. Blank spaced indicated non-detections or lack of observations. The data are taken from Dye et al. (2008a,b).

Table 2. Mid-infrared to radio photometry of SHADES galaxies in the Lockman hole east.

\begin{tabular}{|c|c|c|c|c|c|c|c|}
\hline Name & $15 \mu \mathrm{m}(\mu \mathrm{Jy})$ & $24 \mu \mathrm{m}(\mu \mathrm{Jy})$ & $350 \mu \mathrm{m}$ (mJy) & $850 \mu \mathrm{m}$ (mJy) & $1.1 \mathrm{~mm}(\mathrm{mJy})$ & $1.2 \mathrm{~mm}(\mathrm{mJy})$ & $1.4 \mathrm{GHz}(\mu \mathrm{Jy})$ \\
\hline Lock850.001 & $25 \pm 20$ & $217 \pm 16$ & $24.1 \pm 5.5$ & $8.85_{-1.0}^{+1.0}$ & $4.65_{-0.98}^{+0.97}$ & $3.6 \pm 0.5$ & $78.9 \pm 4.7$ \\
\hline Lock850.002 & $13 \pm 20$ & $545 \pm 31$ & $25.3 \pm 10.3$ & $13.45_{-2.1}^{+2.1}$ & $6.21_{-0.92}^{+1.05}$ & $5.7 \pm 1.0$ & $52.4 \pm 5.6$ \\
\hline Lock850.003 & $208 \pm 20$ & $175 \pm 23$ & $40.5 \pm 6.5$ & $10.95_{-1.9}^{+1.8}$ & $3.01_{-1.06}^{+1.04}$ & $4.6 \pm 0.4$ & $25.8 \pm 4.9$ \\
\hline Lock850.003b & $438 \pm 20$ & $183 \pm 33$ & $40.5 \pm 6.5$ & $10.95_{-1.9}^{+1.8}$ & $3.01_{-1.06}^{+1.04}$ & $4.6 \pm 0.4$ & $35.0 \pm 5.2$ \\
\hline Lock850.004 & $118 \pm 19$ & $179 \pm 68$ & $24.9 \pm 9.1$ & $10.65_{-1.8}^{+1.7}$ & $4.75_{-1.06}^{+0.09}$ & $3.7 \pm 0.4$ & $32.0 \pm 5.1$ \\
\hline Lock850.004b & $59 \pm 19$ & $261 \pm 73$ & & $10.65_{-1.8}^{+1.7}$ & $4.75_{-1.06}^{+0.89}$ & & $73.0 \pm 5.0$ \\
\hline Lock850.005 & $770 \pm 20$ & $58.6 \pm 15.1$ & & $8.15_{-2.1}^{+2.0}$ & & & $<22$ \\
\hline Lock850.006 & $94 \pm 20$ & $75.1 \pm 12.7$ & $38.0 \pm 37.6$ & $6.85_{-1.3}^{+1.3}$ & $2.13_{-1.26}^{+1.12}$ & & $15 \pm 4.8$ \\
\hline Lock850.007 & $35 \pm 19$ & $341 \pm 21$ & & $8.55_{-1.9}^{+1.8}$ & & & $42.6 \pm 5.8$ \\
\hline Lock850.008 & & $481 \pm 25$ & & $5.45_{-1.2}^{+1.1}$ & & & $<22$ \\
\hline Lock850.009 & $87 \pm 20$ & $466 \pm 74$ & & $5.95_{-1.6}^{+1.6}$ & & & $52.6 \pm 4.7$ \\
\hline Lock850.009b & $87 \pm 20$ & $159 \pm 73$ & & $5.95_{-1.6}^{+1.6}$ & & & \\
\hline Lock850.010 & & & $24.1 \pm 12.0$ & $9.15_{-2.9}^{+2.0}$ & & & $25.5 \pm 6.3$ \\
\hline Lock850.010b & & $79.6 \pm 10.8$ & & $9.15_{-2.9}^{+2.7}$ & & & \\
\hline Lock850.011 & $105 \pm 20$ & $112 \pm 57$ & & $6.25_{-1.8}^{+1.7}$ & & & \\
\hline Lock850.012 & $9 \pm 19$ & $263 \pm 19$ & $29.3 \pm 16.0$ & $6.15_{-1.7}^{+1.7}$ & $4.1 \pm 1.3$ & $2.6 \pm 0.4$ & $44.3 \pm 5.1$ \\
\hline Lock850.013 & $47 \pm 20$ & $172 \pm 14$ & & $5.65_{-2.9}^{+2.3}$ & $3.07_{-0.97}^{+0.91}$ & & $<28$ \\
\hline Lock850.014 & $-45 \pm 20$ & $188 \pm 16$ & $41.0 \pm 6.8$ & $7.25_{-1.9}^{+1.8}$ & $1.79_{-1.57}^{+0.97}$ & $3.4 \pm 0.6$ & $37.4 \pm 4.2$ \\
\hline Lock850.015 & $299 \pm 19$ & $353 \pm 20$ & $6.5 \pm 55.7$ & $13.25_{-5.0}^{+4.3}$ & $3.61_{-0.98}^{+1.02}$ & $4.1 \pm 0.7$ & $61.5 \pm 7.6$ \\
\hline Lock850.015b & $299 \pm 19$ & $70.4 \pm 12.1$ & $6.5 \pm 55.7$ & $13.25_{-5.0}^{+4.3}$ & $3.61_{-0.98}^{+1.02}$ & $4.1 \pm 0.7$ & $43.9 \pm 7.8$ \\
\hline Lock850.016 & $321 \pm 19$ & $314 \pm 24$ & $25.7 \pm 15.8$ & $5.85_{-1.9}^{+1.8}$ & & $1.8 \pm 0.5$ & $106 \pm 6$ \\
\hline Lock850.017 & & $239 \pm 18$ & & $4.75_{-1.3}^{+1.3}$ & & & $92.3 \pm 4.5$ \\
\hline Lock850.017b & & $64.2 \pm 26.1$ & & $4.75_{-1.3}^{+1.3}$ & & & Confused \\
\hline Lock850.018 & $40 \pm 19$ & & $7.5 \pm 6.7$ & $6.05_{-2.1}^{+1.9}$ & $5.1 \pm 1.3$ & $3.4 \pm 0.6$ & $29.4 \pm 4.4$ \\
\hline Lock850.019 & & $118 \pm 15$ & & $5.15_{-2.4}^{+2.0}$ & & & $<27$ \\
\hline Lock850.021 & & $97.9 \pm 14.1$ & $16.7 \pm 13.2$ & $4.15_{-2.5}^{+2.0}$ & & $1.6 \pm 0.4$ & $<30$ \\
\hline Lock850.022 & $-15 \pm 20$ & $402 \pm 21$ & $8.7 \pm 15.6$ & $7.55_{-4.2}^{+3.2}$ & & & $<30$ \\
\hline Lock850.023 & & & & $4.35_{-2.4}^{+1.9}$ & & & $<25$ \\
\hline Lock850.024 & $-1 \pm 30$ & $455 \pm 21$ & & $2.75_{-1.2}^{+1.2}$ & & & $28.5 \pm 4.8$ \\
\hline Lock850.026 & $-80 \pm 28$ & $195 \pm 16$ & $12.2 \pm 8.8$ & $5.85_{-2.9}^{+2.4}$ & & & $31.4 \pm 5.2$ \\
\hline Lock850.027 & & $106 \pm 15$ & $3.4 \pm 5.1$ & $5.05_{-1.3}^{+1.3}$ & $5.2 \pm 1.4$ & $3.2 \pm 0.7$ & \\
\hline Lock850.028 & & $252 \pm 14$ & $23.3 \pm 11.7$ & $6.45_{-1.8}^{+1.7}$ & & & $63.0 \pm 8.2$ \\
\hline Lock850.029 & & $111 \pm 14$ & & $6.75_{-2.2}^{+2.0}$ & & & $23.7 \pm 4.9$ \\
\hline Lock850.030 & & $233 \pm 19$ & $38.0 \pm 7.2$ & $4.75_{-1.6}^{+1.5}$ & & $0.4 \pm 0.8$ & $245 \pm 13$ \\
\hline Lock850.031 & & $467 \pm 19$ & & $6.05_{-2.0}^{+1.8}$ & & & $43.0 \pm 4.7$ \\
\hline Lock850.033 & $-71 \pm 20$ & $104 \pm 14$ & $16.5 \pm 8.4$ & $3.85_{-1.1}^{+1.0}$ & & $2.8 \pm 0.6$ & $51.0 \pm 4.3$ \\
\hline Lock850.034 & & $84.9 \pm 16.7$ & & $14.05_{-3.2}^{+3.1}$ & $4.09_{-0.92}^{+0.90}$ & & $58.4 \pm 8.5$ \\
\hline Lock850.035 & $-25 \pm 20$ & $51.0 \pm 12.7$ & & $6.15_{-2.4}^{+2.2}$ & & & $17.4 \pm 5.0$ \\
\hline Lock850.035b & $-53 \pm 20$ & $161 \pm 14$ & & $6.15_{-2.4}^{+2.4}$ & & & \\
\hline Lock850.036 & & $<60$ & & $6.35_{-1.8}^{+1.7}$ & & & $<20$ \\
\hline Lock850.037 & & & & $7.55_{-3.5}^{+2.9}$ & & & $41.8 \pm 8.7$ \\
\hline Lock850.037b & & $250 \pm 17$ & & $7.55_{-3.5}^{+2.9}$ & & & $14.8 \pm 5.4$ \\
\hline
\end{tabular}


S. Serjeant et al.: AGN fraction of submm-selected galaxies \& EBL

Table 2. continued.

\begin{tabular}{|c|c|c|c|c|c|c|c|}
\hline Name & $15 \mu \mathrm{m}(\mu \mathrm{Jy})$ & $24 \mu \mathrm{m}(\mu \mathrm{Jy})$ & $350 \mu \mathrm{m}(\mathrm{mJy})$ & $850 \mu \mathrm{m}(\mathrm{mJy})$ & $1.1 \mathrm{~mm}(\mathrm{mJy})$ & $1.2 \mathrm{~mm}(\mathrm{mJy})$ & $1.4 \mathrm{GHz}(\mu \mathrm{Jy})$ \\
\hline Lock850.038 & $218 \pm 19$ & $260 \pm 16$ & & $4.35_{-27}^{+2.2}$ & & & $24.4 \pm 6.7$ \\
\hline Lock850.039 & & $<60$ & & $6.55_{-2.7}^{+2.2}$ & & & $<20$ \\
\hline Lock850.040 & & $91.9 \pm 15.0$ & & $3.05_{-1.2}^{+1.1}$ & & & $16.2 \pm 4.3$ \\
\hline Lock850.041b & $193 \pm 20$ & $651 \pm 46$ & $10.3 \pm 5.5$ & $3.85_{-1.0}^{+0.9}$ & $4.0 \pm 1.3$ & $2.4 \pm 0.5$ & $22.1 \pm 4.8$ \\
\hline Lock850.041 & $464 \pm 20$ & $475 \pm 37$ & $10.3 \pm 5.5$ & $3.85_{-1.0}^{+0.9}$ & $4.0 \pm 1.3$ & $2.4 \pm 0.5$ & $43.6 \pm 4.7$ \\
\hline Lock850.043 & $140 \pm 19$ & $261 \pm 24$ & & $4.95_{-2.6}^{+2.1}$ & $3.19_{-0.99}^{+1.10}$ & & $25.4 \pm 5.4$ \\
\hline Lock850.043b & $267 \pm 19$ & $456 \pm 35$ & & $4.95_{-2.6}^{+2.1}$ & $3.19_{-0.99}^{+1.10}$ & & $40.8 \pm 5.9$ \\
\hline Lock850.047 & $37 \pm 20$ & $107 \pm 16$ & $16.3 \pm 20.9$ & $3.55_{-2.1}^{+1.7}$ & & & $<22$ \\
\hline Lock850.048 & & $203 \pm 17$ & $16.2 \pm 13.5$ & $5.45_{-2.5}^{+2.1}$ & & $1.6 \pm 0.4$ & $43.7 \pm 10.0$ \\
\hline Lock850.052 & & $310 \pm 35$ & & $3.95_{-2.7}^{+2.2}$ & $2.51_{-1.02}^{+1.03}$ & & $38.7 \pm 8.0$ \\
\hline Lock850.052b & & $561 \pm 86$ & & $3.95_{-2.7}^{+2.2}$ & $2.51_{-1.02}^{+1.03}$ & & Confused \\
\hline Lock850.053 & $-26 \pm 20$ & $168 \pm 15$ & & $4.45_{-2.9}^{+2.3}$ & & & $<21$ \\
\hline Lock850.060 & $4 \pm 20$ & $87.8 \pm 12.0$ & & $3.15_{-2.0}^{+1.7}$ & & & \\
\hline Lock850.063 & $31 \pm 20$ & $236 \pm 17$ & $34.9 \pm 29.1$ & $3.65_{-1.3}^{+1.2}$ & & & $22.6 \pm 4.8$ \\
\hline Lock850.064 & & $425 \pm 25$ & $11.6 \pm 12.4$ & $5.85_{-3.2}^{+2.5}$ & & $1.7 \pm 0.4$ & $45.5 \pm 7.4$ \\
\hline Lock850.066 & & $71.2 \pm 12.1$ & & $4.25_{-2.2}^{+1.9}$ & & & $<21$ \\
\hline Lock850.067 & $59 \pm 20$ & $108 \pm 14$ & & $2.55_{-1.5}^{+1.5}$ & & & $<21$ \\
\hline Lock850.070 & $79 \pm 20$ & $106 \pm 12$ & & $3.85_{-2.5}^{+2.2}$ & & & $21.9 \pm 7.2$ \\
\hline Lock850.071 & & $181 \pm 20$ & & $3.95_{-2.0}^{+1.8}$ & $2.89_{-1.13}^{+1.10}$ & & $95.8 \pm 4.6$ \\
\hline Lock850.073 & $89 \pm 36$ & $278 \pm 19$ & & $3.55_{-2.3}^{+1.9}$ & & & $27.3 \pm 4.8$ \\
\hline Lock850.073b & $143 \pm 61$ & $278 \pm 19$ & & $3.55_{-2.3}^{+1.9}$ & & & $26.7 \pm 4.6$ \\
\hline Lock850.075 & & $147 \pm 17$ & & $4.45_{-2.6}^{+2.3}$ & & & \\
\hline Lock850.076 & $227 \pm 20$ & $592 \pm 26$ & $4.4 \pm 6.7$ & $4.75_{-3.1}^{+2.5}$ & $4.4 \pm 1.4$ & & $48.0 \pm 6.0$ \\
\hline Lock850.077 & $24 \pm 14$ & $51.7 \pm 13.1$ & $41.4 \pm 24.6$ & $3.25_{-1.3}^{+1.1 .2}$ & & & $15.5 \pm 4.4$ \\
\hline Lock850.077b & $2 \pm 15$ & $154 \pm 15$ & & $3.25_{-1.3}^{+1.5}$ & & & $39.5 \pm 7.8$ \\
\hline Lock850.078 & & $85.6 \pm 14.7$ & & $4.55_{-2.7}^{+2.3}$ & & & $<23$ \\
\hline Lock850.079 & $41 \pm 15$ & $292 \pm 18$ & & $3.15_{-1.5}^{+1.3}$ & $2.35_{-1.22}^{+1.12}$ & & $22.41 \pm 4.5$ \\
\hline Lock850.081 & & $3667 \pm 51$ & & $5.35_{-2.3}^{+1.9}$ & & & $55.2 \pm 5.3$ \\
\hline Lock850.083 & & $344 \pm 25$ & & $3.15_{-2.1}^{+2.0}$ & & & $<28$ \\
\hline Lock850.087 & & $399 \pm 22$ & & $3.45_{-17}^{+1.1}$ & & & $84.5 \pm 5.3$ \\
\hline Lock850.100 & & $118 \pm 13$ & & $11.25_{-5.3}^{+4.2}$ & & & $19.8 \pm 6.3$ \\
\hline
\end{tabular}

Notes. The $15 \mu \mathrm{m}$ data are presented for the first time here, and the remaining data are taken from Coppin et al. (2006), Austermann et al. (2010), Ivison et al. (2007), Coppin et al. (2008) and Negrello et al. (2009).

Table 3. Results of the SED fitting, with the parameters defined in the text.

\begin{tabular}{llllllllllll}
\hline \hline Name & $z_{\text {phot }}$ & $\delta z_{\text {phot,min }}$ & $\delta z_{\text {phot,max }}$ & $\chi_{v}^{2}$ & $N_{\text {D.O.F. }}$ & Dust & Age & $\Theta$ & $\theta_{\text {view }}$ & $f_{\text {AGN }}$ & Notes \\
\hline LOCK850.001 & 5.30 & 1.10 & 0.46 & 0.98 & 8 & SMC & 50 & 1.6 & 35 & 0.73 & \\
LOCK850.002 & 3.68 & 0.34 & 0.24 & 4.15 & 8 & LMC & 50 & 1.2 & 5 & 0.77 & I \\
LOCK850.003 & 3.80 & 0.22 & 0.20 & 6.67 & 8 & SMC & 50 & 2.0 & 0 & 0.70 & \\
LOCK850.003B & 6.56 & 1.14 & 0.44 & 7.15 & 8 & MW & 600 & 2.2 & 35 & 0.98 & D, C \\
LOCK850.004 & 3.76 & 0.56 & 2.90 & 2.77 & 9 & SMC & 50 & 1.6 & 45 & 0.08 & $\mathrm{U}$ \\
LOCK850.004B & 6.54 & 1.88 & 0.46 & 3.54 & 7 & SMC & 400 & 1.8 & 45 & 0.42 & $\mathrm{U}$ \\
LOCK850.005 & 6.14 & 0.60 & 0.44 & 10.02 & 6 & SMC & 10 & 1.2 & 30 & 0.86 & $\mathrm{C}$ \\
LOCK850.006 & 5.58 & 1.94 & 1.41 & 3.31 & 8 & SMC & 70 & 2.0 & 42.5 & 0.20 & $\mathrm{I}$ \\
LOCK850.007 & 2.56 & 0.60 & 4.44 & 0 & -3 & SMC & 50 & 0.3 & 10 & 0.18 & $\mathrm{I}$ \\
LOCK850.008 & 0.22 & 0.06 & 6.78 & 0 & -4 & SMC & 200 & 0.3 & 0 & 0.00 & $\mathrm{I}$ \\
LOCK850.009 & 2.30 & 0.54 & 0.70 & 2.36 & 6 & SMC & 400 & 1.4 & 32.5 & 0.56 & $\mathrm{U}, \mathrm{X}$ \\
LOCK850.009B & 1.18 & 0.46 & 0.42 & 2.10 & 8 & MW & 600 & 1.8 & 0 & 0.00 & $\mathrm{U}$ \\
LOCK850.010B & 3.28 & 0.68 & 0.52 & 3.05 & 5 & SMC & 50 & 1.2 & 90 & 0.00 & \\
LOCK850.011 & 1.24 & 0.32 & 0.40 & 2.38 & 6 & MW & 500 & 1.4 & 15 & 0.55 & $\mathrm{X}$ \\
LOCK850.012 & 2.26 & 0.36 & 1.45 & 2.03 & 7 & SMC & 200 & 1.6 & 0 & 0.37 & $\mathrm{I}$ \\
LOCK850.013 & 0.50 & 0.26 & 0.20 & 2.36 & 7 & SMC & 600 & 2.0 & 7.5 & 0.09 & $\mathrm{X}$ \\
LOCK850.014 & 3.06 & 0.44 & 0.36 & 3.72 & 7 & SMC & 50 & 1.8 & 50 & 0.01 & \\
LOCK850.015 & 1.40 & 0.84 & 0.40 & 3.79 & 8 & SMC & 400 & 0.3 & 27.5 & 0.11 & \\
LOCK850.015B & 1.36 & 0.18 & 0.16 & 2.54 & 8 & SMC & 500 & 0.4 & 7.5 & 0.32 & $\mathrm{D}$ \\
\hline
\end{tabular}


Table 3. continued.

\begin{tabular}{|c|c|c|c|c|c|c|c|c|c|c|c|}
\hline Name & $z_{\text {phot }}$ & $\delta z_{\text {phot,min }}$ & $\delta z_{\text {phot,max }}$ & $\chi_{v}^{2}$ & $N_{\text {D.O.F. }}$ & Dust & Age & $\Theta$ & $\theta_{\text {view }}$ & $f_{\mathrm{AGN}}$ & Notes \\
\hline LOCK850.016 & 6.58 & 0.54 & 0.42 & 5.22 & 7 & SMC & 10 & 1.2 & 40 & 0.44 & \\
\hline LOCK850.017 & 3.48 & 1.18 & 0.34 & 2.19 & 5 & SMC & 100 & 2.6 & 7.5 & 0.50 & $\mathrm{U}$ \\
\hline LOCK850.017B & 4.20 & 0.68 & 1.98 & 1.72 & 5 & LMC & 50 & 1.4 & 0 & 0.65 & $\mathrm{U}$ \\
\hline LOCK850.019 & 3.94 & 0.24 & 1.92 & 1.11 & 5 & SMC & 50 & 2.0 & 5 & 0.69 & I \\
\hline LOCK850.021 & 0.86 & 0.36 & 6.14 & 0.91 & 6 & SMC & 500 & 0.4 & 17.5 & 0.15 & I \\
\hline LOCK850.022 & 3.10 & 0.90 & 0.40 & 2.24 & 6 & MW & 600 & 5.0 & 0 & 0.96 & \\
\hline LOCK850.024 & 2.72 & 0.70 & 0.56 & 2.26 & 5 & SMC & 30 & 1.8 & 0 & 0.24 & \\
\hline LOCK850.026 & 3.48 & 1.24 & 0.46 & 3.23 & 6 & SMC & 30 & 1.6 & 0 & 0.74 & I \\
\hline LOCK850.027 & 1.34 & 0.18 & 0.34 & 2.97 & 6 & MW & 600 & 2.2 & 0 & 0.66 & \\
\hline LOCK850.028 & 3.66 & 0.58 & 0.20 & 1.63 & 6 & SMC & 50 & 2.2 & 7.5 & 0.50 & \\
\hline LOCK850.029 & 4.44 & 3.38 & 2.56 & 0.23 & 5 & SMC & 100 & 1.8 & 0 & 0.96 & I \\
\hline LOCK850.030 & 2.70 & 1.04 & 0.53 & 2.03 & 6 & SMC & 50 & 0.9 & 50 & 0.00 & \\
\hline LOCK850.031 & 1.12 & 0.50 & 1.40 & 2.08 & 5 & SMC & 400 & 0.7 & 17.5 & 0.46 & $X$ \\
\hline LOCK850.033 & 2.78 & 0.66 & 0.70 & 4.75 & 6 & LMC & 70 & 1.2 & 50 & 0.00 & \\
\hline LOCK850.034 & 4.14 & 0.45 & 2.02 & 3.19 & 6 & LMC & 50 & 1.2 & 90 & 0.00 & \\
\hline LOCK850.035 & 5.24 & 3.26 & 1.76 & 0.72 & 5 & SMC & 70 & 2.0 & 7.5 & 0.94 & I \\
\hline LOCK850.035B & 2.20 & 0.28 & 0.78 & 4.53 & 5 & MW & 600 & 2.2 & 0 & 0.66 & $\mathrm{D}$ \\
\hline LOCK850.036 & 0.92 & 0.54 & 6.08 & 0 & -4 & $\mathrm{SMC}$ & 200 & 0.3 & 0 & 0.00 & I \\
\hline LOCK850.037B & 1.26 & 0.46 & 1.00 & 1.84 & 5 & SMC & 300 & 1.4 & 22.5 & 0.38 & $X$ \\
\hline LOCK850.038 & 1.36 & 0.30 & 0.30 & 1.11 & 6 & MW & 300 & 2.4 & 20 & 0.30 & \\
\hline LOCK850.039 & 0.92 & 0.74 & 6.08 & 0 & -4 & SMC & 200 & 0.3 & 0 & 0.00 & I \\
\hline LOCK850.040 & 2.24 & 1.64 & 0.34 & 2.02 & 5 & MW & 400 & 1.8 & 0 & 0.10 & $X$ \\
\hline LOCK850.041B & 2.44 & 0.94 & 1.38 & 3.38 & 6 & LMC & 50 & 2.2 & 40 & 0.31 & \\
\hline LOCK850.041 & 1.60 & 0.44 & 0.10 & 1.61 & 7 & LMC & 200 & 2.4 & 7.5 & 0.31 & \\
\hline LOCK850.043 & 1.38 & 0.70 & 0.66 & 2.03 & 7 & SMC & 200 & 1.6 & 27.5 & 0.42 & $\mathrm{U}, \mathrm{X}$ \\
\hline LOCK850.043B & 1.60 & 0.58 & 0.40 & 2.21 & 7 & SMC & 100 & 2.0 & 0 & 0.22 & $\mathrm{U}, \mathrm{X}$ \\
\hline LOCK850.047 & 3.82 & 0.20 & 0.40 & 1.26 & 7 & LMC & 30 & 3.0 & 37.5 & 0.40 & \\
\hline LOCK850.048 & 4.18 & 0.86 & 2.16 & 1.70 & 6 & SMC & 10 & 1.6 & 90 & 0.15 & \\
\hline LOCK850.052 & 1.42 & 0.32 & 0.76 & 3.77 & 6 & $\mathrm{SMC}$ & 600 & 1.0 & 0 & 0.73 & $\mathrm{U}$ \\
\hline LOCK850.052B & 3.64 & 0.68 & 0.58 & 5.73 & 5 & SMC & 600 & 1.4 & 5 & 0.97 & $\mathrm{U}$ \\
\hline LOCK850.053 & 2.22 & 0.40 & 0.64 & 2.25 & 5 & MW & 600 & 2.4 & 0 & 0.64 & $\mathrm{X}$ \\
\hline LOCK850.060 & 2.46 & 0.90 & 2.90 & 2.27 & 6 & MW & 600 & 1.2 & 0 & 0.64 & \\
\hline LOCK850.063 & 4.98 & 0.88 & 0.88 & 0.42 & 7 & MW & 600 & 2.6 & 32.5 & 0.96 & I \\
\hline LOCK850.064 & 2.10 & 1.41 & 1.28 & 0.98 & 6 & LMC & 50 & 2.2 & 0 & 0.27 & I \\
\hline LOCK850.066 & 3.72 & 0.00 & 1.30 & 0.61 & 5 & SMC & 30 & 0.7 & 50 & 0.03 & \\
\hline LOCK850.067 & 7.00 & 0.72 & 0.00 & 3.33 & 6 & MW & 400 & 3.0 & 40 & 0.83 & \\
\hline LOCK850.070 & 0.62 & 0.22 & 0.36 & 0.61 & 6 & MW & 500 & 1.8 & 22.5 & 0.05 & $\mathrm{X}$ \\
\hline LOCK850.071 & 2.24 & 1.48 & 0.88 & 1.91 & 6 & MW & 600 & 1.8 & 0 & 0.67 & $\mathrm{X}$ \\
\hline LOCK850.073 & 1.72 & 0.96 & 1.88 & 0.64 & 6 & LMC & 100 & 2.0 & 50 & 0.00 & \\
\hline LOCK850.073B & 2.26 & 1.52 & 1.30 & 1.17 & 4 & MW & 400 & 2.4 & 37.5 & 0.45 & D \\
\hline LOCK850.075 & 1.28 & 0.00 & 2.54 & 1.30 & 3 & MW & 400 & 2.2 & 45 & 0.42 & \\
\hline LOCK850.076 & 0.48 & 0.14 & 0.12 & 2.22 & 8 & SMC & 600 & 1.0 & 7.5 & 0.06 & $\mathrm{X}$ ? \\
\hline LOCK850.077 & 3.20 & 2.62 & 0.48 & 2.77 & 7 & SMC & 50 & 1.2 & 40 & 0.00 & $\mathrm{U}$ \\
\hline LOCK850.077B & 3.74 & 0.32 & 0.34 & 5.01 & 4 & LMC & 500 & 1.6 & 7.5 & 0.69 & $\mathrm{U}$ \\
\hline LOCK850.078 & 3.38 & 0.00 & 2.64 & 0.57 & 1 & SMC & 50 & 1.4 & 50 & 0.02 & \\
\hline LOCK850.079 & 1.96 & 0.24 & 0.22 & 2.50 & 7 & MW & 600 & 1.2 & 22.5 & 0.57 & \\
\hline LOCK850.081 & 3.18 & 1.04 & 0.92 & 0.83 & 5 & SMC & 400 & 2.0 & 42.5 & 0.84 & I \\
\hline LOCK850.083 & 0.44 & 0.08 & 0.06 & 1.89 & 7 & LMC & 600 & 1.4 & 0 & 0.00 & \\
\hline LOCK850.087 & 2.54 & 0.82 & 0.82 & 0.50 & 5 & SMC & 300 & 1.4 & 0 & 0.57 & \\
\hline LOCK850.100 & 3.66 & 3.40 & 2.60 & 1.85 & 1 & SMC & 200 & 1.6 & 55 & 0.08 & \\
\hline
\end{tabular}

Notes. All parameters are dimensionless except the age which is in Myr. In the final column, "C" notes possibly contaminated flux at $15 \mu \mathrm{m}$, "D" notes a depreciated identification, "I" insufficient data to determine the AGN bolometric fraction, "U" an uncertain identification, and "X" a far-infrared excess.

The small background contribution at $1100 \mu \mathrm{m}$ is in stark contrast to our earlier results for the submm backgrounds (Serjeant et al. 2008) in which we found the majority of the $450 \mu \mathrm{m}$ background is attributable to the $24 \mu \mathrm{m}$-selected population, and to claims from the Balloon-Borne Large Area Submm Telescope (BLAST) data that the mid-infrared population is responsible for all the 250-500 $\mu \mathrm{m}$ background (Devlin et al. 2009; Marsden et al. 2009). Taken in combination with the results in Serjeant et al. (2008), our results suggest strongly that other populations undetected by AKARI or Spitzer must dominate the $850 \mu \mathrm{m}$ and $\mathrm{mm}$-wave background.

Not all our sample has sufficient mid-infrared data to distinguish starburst and AGN mid-infrared contributions, but Table 3 gives the results where available. In total 41 SHADES galaxies have sufficient data for this constraint, not counting multiple identifications. Of these, we find that our models cannot reproduce the far-infrared luminosity in 10 unambiguous identifications (LOCK850.013, 031, 037B, 038, 040, 053, 060, 
S. Serjeant et al.: AGN fraction of submm-selected galaxies \& EBL
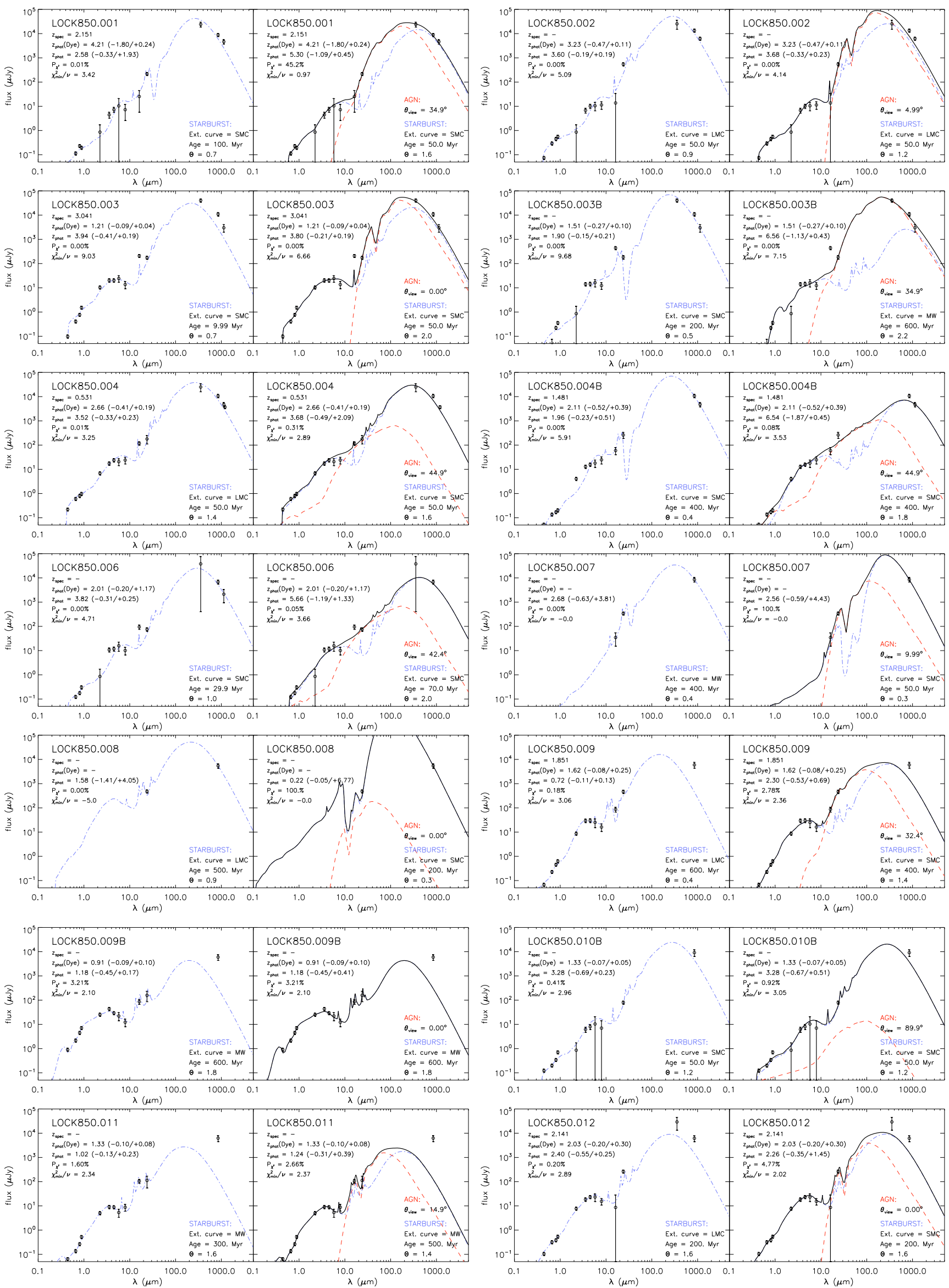

Fig. 6. SED fits to submm-selected SHADES galaxies in the Lockman hole east, using models from Takagi et al. (2003, 2004) and Efstathiou \& Rowan-Robinson (1995). 
A\&A 514, A10 (2010)
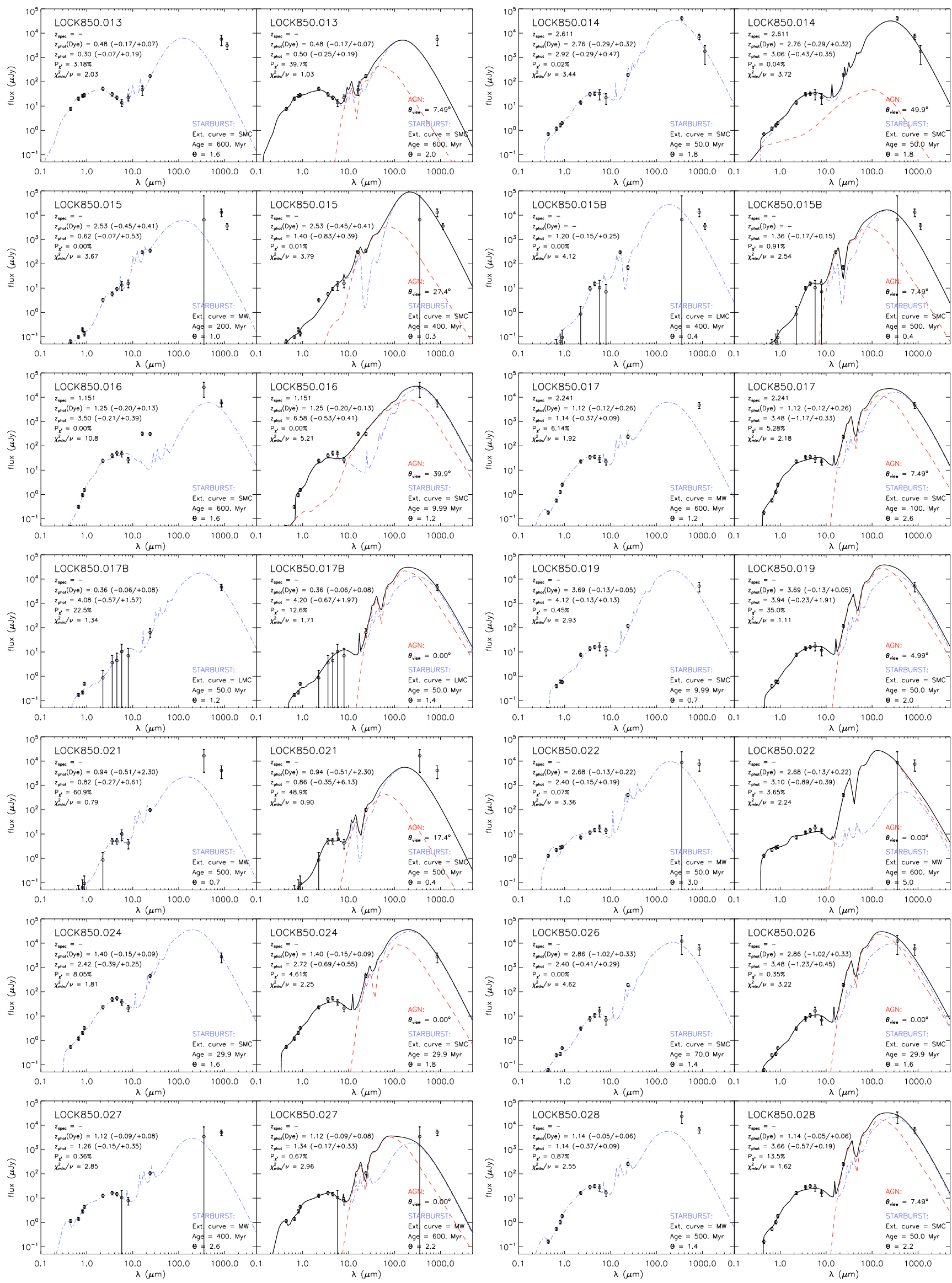

Fig. 7. SED fits to submm-selected SHADES galaxies in the Lockman hole east, using models from Takagi et al. (2003, 2004) and Efstathiou \& Rowan-Robinson (1995). 
S. Serjeant et al.: AGN fraction of submm-selected galaxies \& EBL
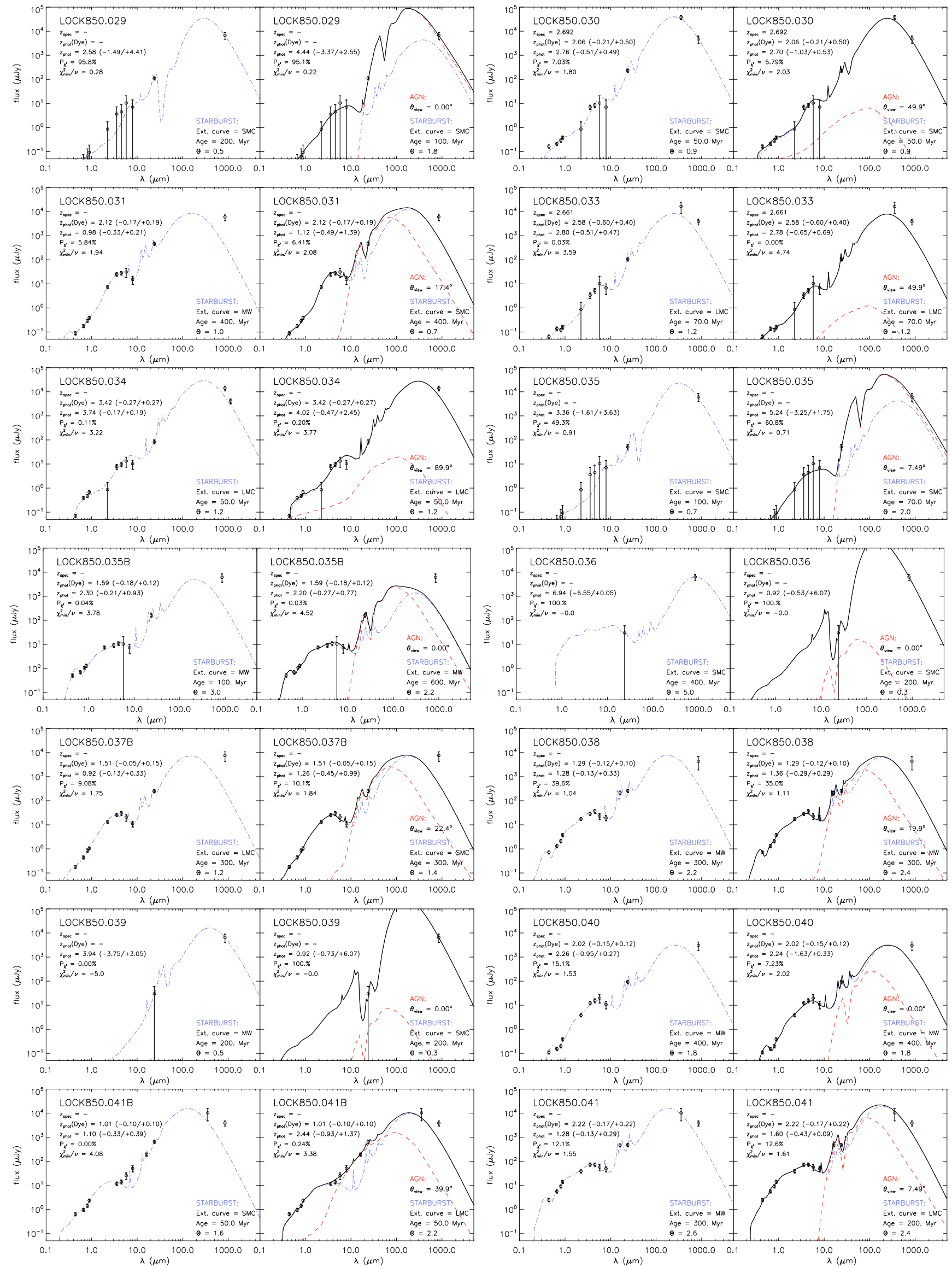

Fig. 8. SED fits to submm-selected SHADES galaxies in the Lockman hole east, using models from Takagi et al. (2003, 2004) and Efstathiou \& Rowan-Robinson (1995). 

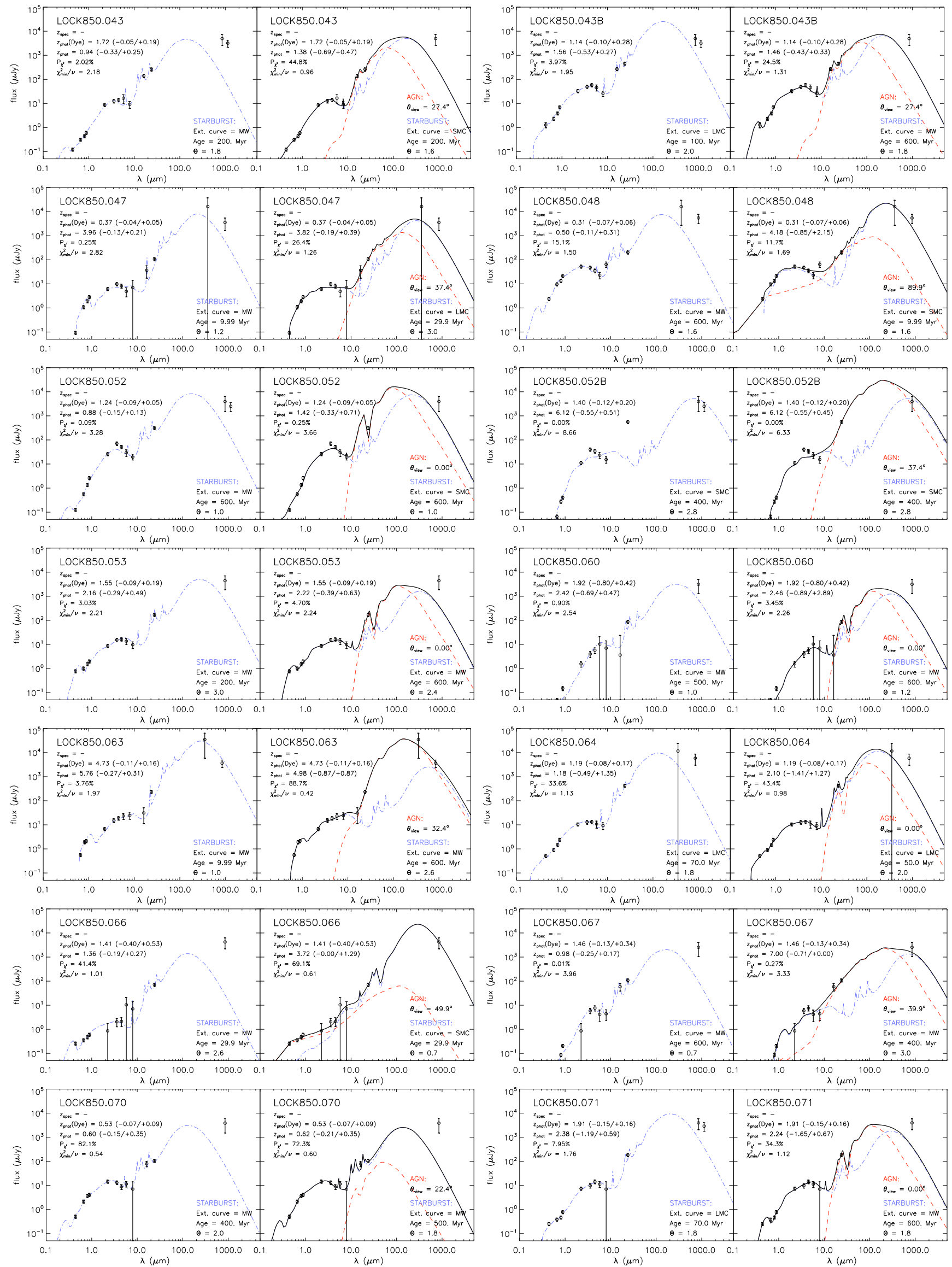

Fig. 9. SED fits to submm-selected SHADES galaxies in the Lockman hole east, using models from Takagi et al. (2003, 2004) and Efstathiou \& Rowan-Robinson (1995). 

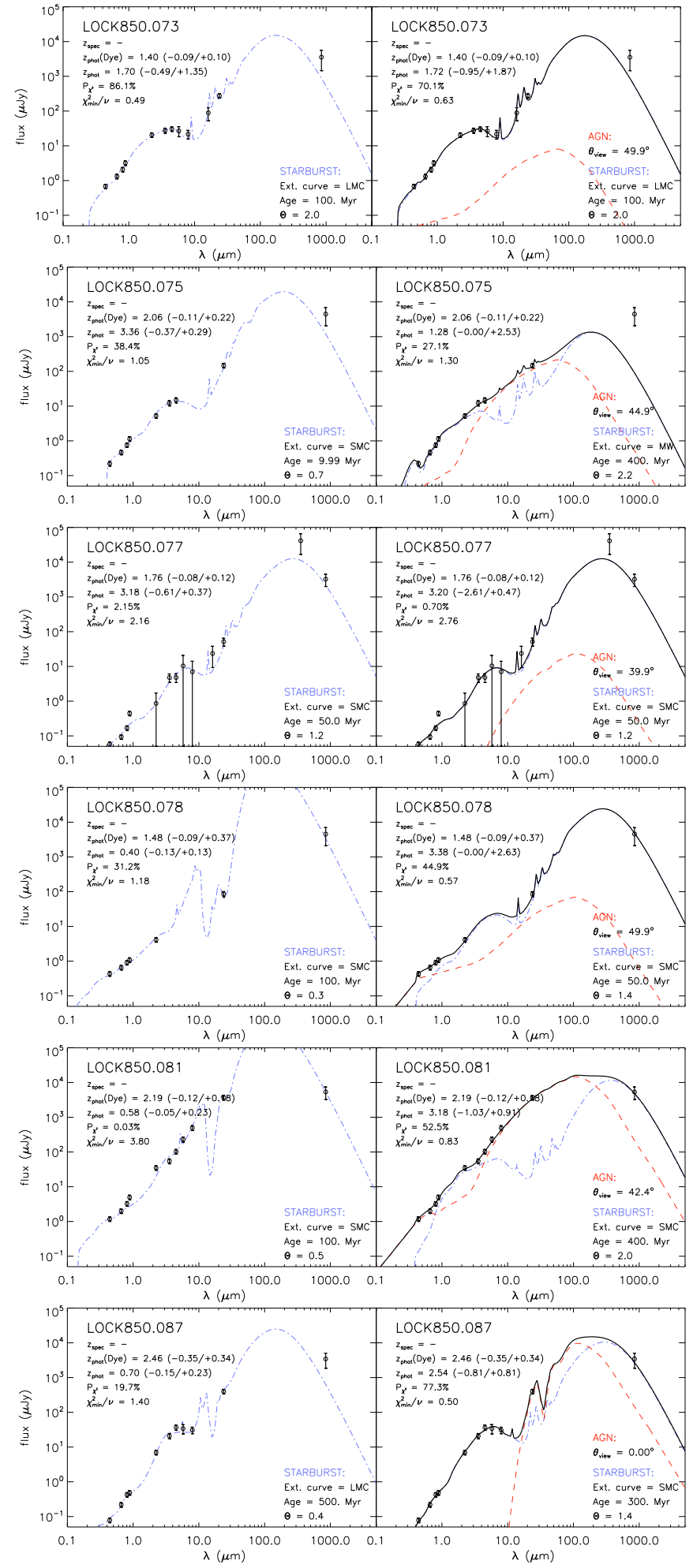
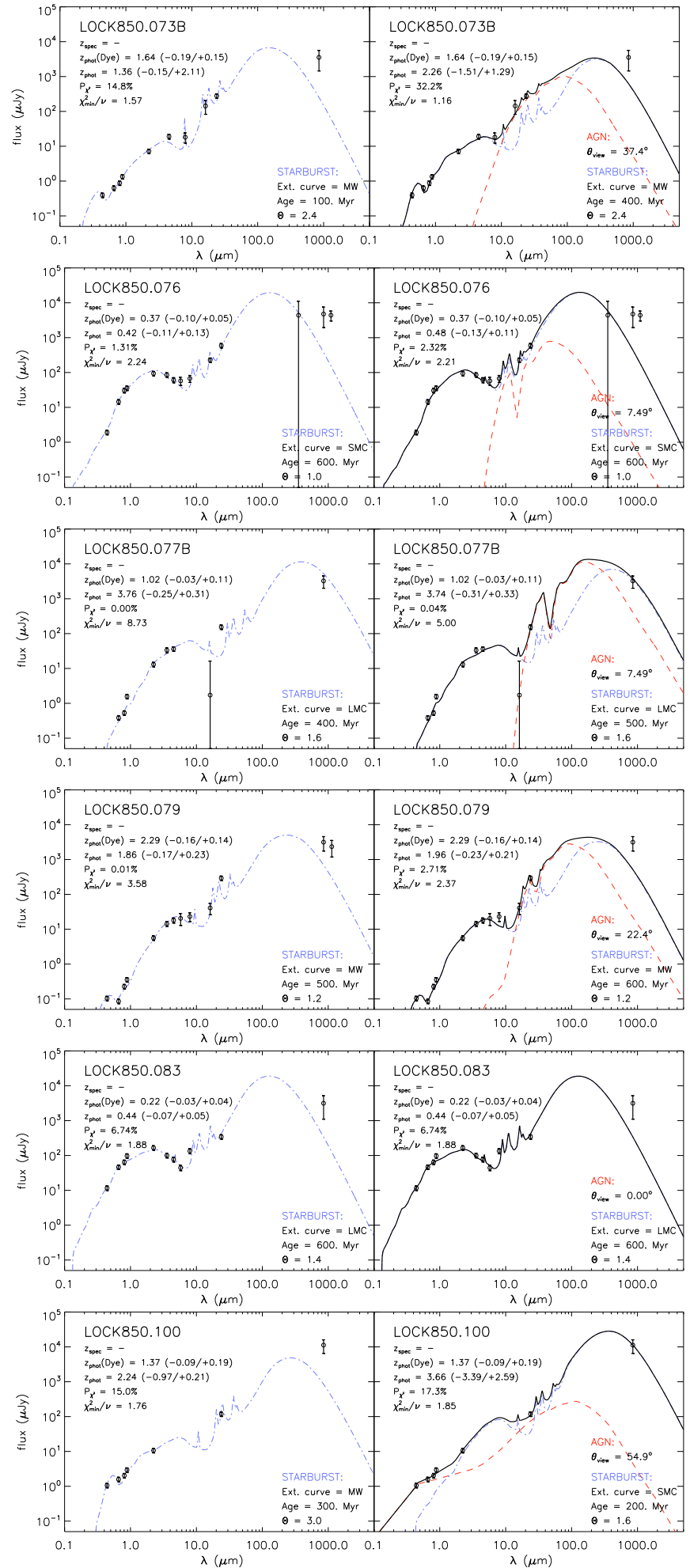

Fig. 10. SED fits to submm-selected SHADES galaxies in the Lockman hole east, using models from Takagi et al. (2003, 2004) and Efstathiou \& Rowan-Robinson (1995).

070, 071, 075) and in two further ambiguous identifications (LOCK850.009, 043). One possibility is that the spectral energy distributions have an additional cool cirrus component not accounted for in the models, which would further reduce an AGN bolometric contribution. Another possibility is that the identifications are wrong, and the submm emission comes from objects with larger far-infrared to optical/near-infrared luminosity ratios. Of the remaining (apparently) unambiguously-identified objects, 12 have AGN fractions $>0.3$ (LOCK850.001, 003, 016, $022,027,028,041,047,052,067,079,087)$, while 14 have starburst fractions $>0.7$ (LOCK850.010B, 014, 015, 024, 030, 033, 034, 048, 066, 073, 076, 078, 083, 100). For LOCK850.004 and LOCK850.077 the two candidate identifications result in one starburst $\left(f_{\mathrm{AGN}}<0.3\right)$ and one $\mathrm{AGN}\left(f_{\mathrm{AGN}}>0.3\right)$ interpretation 
for each galaxy. Finally two further cases merit individual attention. In LOCK850.060, the addition of the AGN component created a worse fit in the submm; we opt to attribute greater weight to this long-wavelength data point and include this galaxy among the starburst. In LOCK850.076 there is additional mmwave flux, which we attribute to errors in the deboosting the submm or mm-wave data in this individual object; this galaxy is also counted among the starbursts.

In summary therefore, considering only those galaxies in which sufficient data is available, we have 12 galaxies with an unknown far-infrared excess relative to our models, 12 with AGN fractions $f_{\mathrm{AGN}}>0.3,16$ starburst-dominated $\left(f_{\mathrm{AGN}}<0.3\right)$. Note that we previously found that AGN bolometric fractions above 0.3 cannot be reliably measured in this broad-band fitting (Negrello et al. 2009). If we exclude the poorly-fit SEDs, we find that $(43 \pm 9) \%$ of our submm-selected galaxies have $f_{\text {AGN }}>0.3$; alternatively, if we conservatively include the galaxies with far-infrared excesses as starburst-dominated, we find $(30 \pm 7) \%$ have AGN bolometric contributions above this threshold. We therefore treat the $1 \sigma$ range to be $(23-52) \%$. Pope et al. (2008) used mid-infrared spectroscopy of submm-selected galaxies and found that a fraction $(15 \pm 10) \%$ (i.e. $2 / 13$ galaxies in their sample) had an active nucleus which contributes significantly to the mid-infrared. This is slightly lower than found in our study, though only marginally inconsistent given the small number statistics in both studies.

Eight of our SHADES galaxies have been identified as having anomalous 610-1400 MHz spectral indices (Ibar et al., in preparation): LOCK850.001, 015, 018, 022, 024, 033, 040, 087. Of these, LOCK850.001, 024 and 033 have flat radio spectra $\left(-\mathrm{d} \ln S_{v} / \mathrm{d} \ln v<0.5\right)$, while the remainder have unusually steep spectra, consistent with synchrotron ageing of electrons in the radio lobes from active nuclei. We have identified three of these eight securely as having AGN bolometric contributions $f_{\mathrm{AGN}}>0.3$, and three with $f_{\mathrm{AGN}}<0.3$. This suggests that evidence of AGN in the radio is not necessarily an indicator of bolometrically-dominant active nuclei.

The high star formation rates (several hundred $M_{\odot} /$ year, e.g. Hughes et al. 1998) and low number density of submmselected galaxies (e.g. Scott et al. 2006; Coppin et al. 2006) suggest that submm galaxies are short-lived. Indeed the steep number counts of submm-selected galaxies necessarily imples that we sample submm galaxies at around their peak phase of far-infrared luminosity. Even a slowly-varying monochromatic luminosity will induce this effect: in the models of Takagi et al. (2003, 2004), the submm luminosity varies by around a factor of 2-3 over a timescale of $\sim 200$ Myr. Denoting this for simplicity as $S_{v}(t) \propto \mathrm{e}^{-0.5(\tau / \sigma)^{2}}$, where $\tau$ measures the secular evolution and $\sigma \simeq(200 / 2.35) \mathrm{Myr}$ is the timescale of the variation, we find that a power-law differential source counts of $\mathrm{d} N / \mathrm{d} S \propto S^{-\alpha}$ gives an observed age distribution in a flux-limited sample of

$\operatorname{Pr}(\tau) \propto \mathrm{e}^{-\left(\alpha+\frac{1}{2}\right)\left(\frac{\tau}{\sigma}\right)^{2}}=\mathrm{e}^{-\frac{1}{2}\left(\frac{\tau}{\sigma^{\prime}}\right)^{2}}$

where $\left(\sigma^{\prime}\right)^{2}=\sigma^{2} /(2 \alpha+1)$. This is a far shorter timescale than the intrinsic variation, given the observed counts slope of $\alpha \simeq 3.5-4$ (Coppin et al. 2006).

There have been suggestions from numerical simulations (e.g. di Matteo et al. 2005) that feedback from active nuclei is capable of truncating star formation activity. However quasars also have star-forming hosts (e.g. Serjeant \& Hatziminaoglou 2009) which may pose challenges for models in which star formation is truncated too abruptly by active nuclei (e.g. Narayanan et al. 2010). Therefore we suggest that the energy input from active nuclei through quasar-mode or radio-mode feedback does not immediately truncate star formation, but rather suppresses it on the same timescales as the quasar lifetime itself. We may also find that star-forming far-infrared-luminous populations selected at shorter wavelengths than SHADES have warmer colour temperatures (e.g. Blain et al. 2003) and higher AGN bolometric contributions. In this interpretation, these would be later phases in the co-evolution of active nucleus and starburst. This would also be consistent with the observation that the $K$-band Hubble diagram of hyperluminous starbursts is tight when the hyperluminous galaxies are selected at $60 \mu \mathrm{m}$ (Serjeant et al. 2003b), but has a much higher dispersion when the hyperluminous galaxies are selected at submm-wavelengths (e.g. Smail et al. 2004).

Finally, our models predict submm-selected galaxies have fluxes of $\simeq 1-10 \mathrm{mJy}$ at an observed frame wavelength of $70 \mu \mathrm{m}$. This was beyond the capabilities of both AKARI and Spitzer for direct detections, but is consistent with the Spitzer stacking analysis detection by Dye et al. (2007) of (3.63 \pm 0.77$) \mathrm{mJy}$. Direct detections at this depth may be accessible to Herschel at shorter wavelengths, and would probe the hot dust components in submm-selected galaxies, currently not strongly constrained.

Acknowledgements. This research is based on observations with AKARI, a JAXA project with the participation of ESA. This work was funded in part by STFC (grant PP/D002400/1), the Royal Society (2006/R4-IJP) and the Sasakawa Foundation (3108). J.S.D. thanks the Royal Society for a Wolfson Research Merit Award. M.I. was supported by the Korea Science and Engineering Foundation(KOSEF) grant No. 2009-0063616, funded by the Korea government(MEST). S.K. were supported by the Basic Science Research Programme through the National Research Foundation of Korea (NRF) funded by the Ministry of Education, Science and Technology 2009-0066892. We thank the anonymous referee for helpful comments.

\section{References}

Alexander, D. M., Bauer, F. E., Chapman, S. C., et al. 2005, ApJ, 632, 736 Austermann, J. E., Dunlop, J. S., Perera, T. A., et al. 2010, MNRAS, 401, 160 Aretxaga, I., Terlevich, R. J., \& Boyle, B. J. 1998, MNRAS, 296, 643 Aretxaga, I., Hughes, D. H., Coppin, K., et al. 2007, MNRAS, 379, 1571 Barger, A. J., Cowie, L. L., Sanders, D. B., et al. 1998, Nature, 394, 248 Baugh, C. M., Lacey, C. G., Frenk, C. S., et al. 2005, MNRAS, 356, 1191 Bertoldi, F., \& Cox, P. 2002, A\&A, 384, L11

Bertoldi, F., Carilli, C. L., Cox, P., et al. 2003, A\&A, 406, L55 Blain, A. W., Barnard, V. E., \& Chapman, S. C. 2003, MNRAS, 338, 733 Brotherton, M. S., van Breugel, W., Stanford, S. A., et al. 1999, ApJ, 520, L87 Carilli, C. L., Bertoldi, F., Rupen, M. P., et al. 2001, ApJ, 555, 625 Clements, D. L., Vaccari, M., Babbedge, T., et al. 2008, MNRAS, 387, 247 Coppin, K., Halpern, M., Scott, D., et al. 2004, MNRAS, 354, 193 Coppin, K., Chapin, E. L., Mortier, A. M. J., et al. 2006, MNRAS, 372, 1621 Coppin, K., Halpern, M., Scott, D., et al. 2008, MNRAS, 384, 1597 Devlin, M. J., Ade, P. A. R., Aretxaga, I., et al. 2009, Nature, 458, 737 di Matteo, T., Springel, V., \& Hernquist, L. 2005, Nature, 433, 604 Dye, S., Eales, S. A., Ashby, M. L. N., et al. 2007, MNRAS, 375, 725 Dey, A., Soifer, B. T., Desai, V., et al. 2008a, ApJ, 677, 943 Dye, S., Eales, S. A., Aretxaga, I., et al. 2008b, MNRAS, 386, 1107 Eddington, A. S. 1913, MNRAS, 73, 359

Efstathiou, A., \& Rowan-Robinson, M. 1995, MNRAS, 273, 649 Elbaz, D., Cesarsky, C. J., Fadda, D., et al. 1999, A\&A, 351, L37 Fazio, G. G., Hora, J. L., Allen, L. E., et al. 2004, ApJS, 154, 10 Ferrarese, L., \& Merritt, S. 2000, ApJ, 539, L9

Genzel, R., \& Cezarsky, C. 2005, ARA\&A, 38, 761

Gispert, R., Lagache, G., \& Puget, J. L. 2000, A\&A, 360, 1 Granato, G. L., Silva, L., Lapi, A., et al. 2006, MNRAS, 368, L72 Greve, T. R., Weiss, A., Walter, F., et al. 2009, [arXiv:0904 .0028] Hopwood, R. H., et al. 2009, ApJL, submitted

Hughes, D. H., Dunlop, J. S., \& Rawlings, S. 1997, MNRAS, 289, 766 Hughes, D. H., Serjeant, S., Dunlop, J., et al. 1998, Nature, 394, 241 Isaak, K. G., Priddey, R. S., McMahon, R. G., et al. 2002, MNRAS, 329, 149 Ivison, R. J., Greve, T. R., Dunlop, J. S., et al. 2007, MNRAS, 380, 199 Lacey, C. G., Baugh, C. M., Frenk, C. S., et al. 2008, MNRAS, 385, 1155 Lehnert, M. D., Heckman, T. M., Chambers, K. C., \& Miley, G. K. 1992, ApJ, 393, 68

Lutz, D., Sturm, E., Tacconi, L. J., et al. 2008, ApJ, 684, 853 
S. Serjeant et al.: AGN fraction of submm-selected galaxies \& EBL

Magorrian, J., Tremaine, S., Richstone, D., et al. 1998, AJ, 115, 2285 Marsden, G., Ade, P. A. R., Bock, J. J., et al. 2009, ApJ, 707, 1729

Martínez-Sansigre, A., Rawlings, S., Lacy, M., et al. 2005, Nature, 436, 666

Menéndez-Delmestre, K., Blain, A. W., Smail, I., et al. 2009, ApJ, 699, 667

Mortier, A. M. J., Serjeant, S., Dunlop, J. S., et al. 2005, MNRAS, 363, 563

Mullaney, J. R., Alexander, D. M., Huynh, M., Goulding, A. D., \& Frayer, D. 2010, MNRAS, 401, 995

Narayanan, D., Hayward, C. C., Cox, T. J., et al. 2010, MNRAS, 401, 1613

Negrello, M., Serjeant, S., Pearson, C., et al. 2009, MNRAS, 394, 375

Oliver, S., Mann, R. G., Carballo, R., et al. 2002, MNRAS, 332, 536

Omont, A., Cox, P., Bertoldi, F., et al. 2001, A\&A, 374, 371

Omont, A., Beelen, A., Bertoldi, F., et al. 2003, A\&A, 398, 857

Peacock, J. A. 1999, Cosmological Physics (Cambridge University Press)

Peacock, J. A., Rowan-Robinson, M., Blain, A. W., et al. 2000, MNRAS, 318, 535

Pearson, C. P. 2005, MNRAS, 358, 1417

Pearson, C. P., Serjeant, S., Negrello, M., et al. 2010, A\&A, 514, A9

Pope, A., Chary, R.-R., Alexander, D. M., et al. 2008, ApJ, 675, 1171

Priddey, R. S., Isaak, K. G., McMahon, R. G., \& Omont, A. 2003a, MNRAS, 339,1183
Priddey, R. S., Isaak, K. G., McMahon, R. G., Robson, E. I., \& Pearson, C. P. 2003b, MNRAS, 344, L74

Rieke, G. H., Young, E. T., Engelbracht, C. W., et al. 2004, ApJS, 154, 25

Rodighiero, G., Lari, C., Fadda, D., et al. 2004, A\&A, 427, 773

Scott, S. E., Dunlop, J. S., \& Serjeant, S. 2006, MNRAS, 370, 1057

Scott, K. S., Austermann, J. E., Perera, T. A., et al. 2008, MNRAS, 385, 2225

Serjeant, S., \& Hatziminaoglou, E. 2009, MNRAS, 397, 265

Serjeant, S., Dunlop, J. S., Mann, R. G., et al. 2003a, MNRAS, 344, 887

Serjeant, S., Farrah, D., Geach, J., et al. 2003b, MNRAS, 346, L51

Serjeant, S., Mortier, A. M. J., Ivison, R. J., et al. 2004, ApJS, 154, 118

Serjeant, S., Dye, S., Mortier, A., et al. 2008, MNRAS, 386, 1907

Shi, Y., Rieke, G. H., Ogle, P., et al. 2009, ApJ, 703, 1107

Smail, I., Ivison, R. J., \& Blain, A. W. 1997, ApJ, 490, L5

Smail, I., Chapman, S. C., Blain, A. W., \& Ivison, R. J. 2004, ApJ, 616, 71

Takagi, T., Arimoto, N., \& Hanami, H. 2003, MNRAS, 340, 813

Takagi, T., Hanami, H., \& Arimoto, N. 2004, MNRAS, 355, 424

Takagi, T., Mortier, A. M. J., Shimasaku, K., et al. 2007, MNRAS, 381, 1154

Veilleux, S., Rupke, D. S. N., Kim, D.-C., et al. 2009, ApJS, 182, 628

Wang, W.-H., Barger, A. J., \& Cowie, L. L. 2007, ApJ, 690, 319

Willott, C., Rawlings, S., \& Grimes, J. A. 2003, ApJ, 598, 909

Yun, M. S., Aretxaga, I., Ashby, M. L. N., et al. 2008, MNRAS, 389, 333 